\title{
MULTISCALE TAILORED FINITE POINT METHOD FOR SECOND ORDER ELLIPTIC EQUATIONS WITH ROUGH OR HIGHLY OSCILLATORY COEFFICIENTS*
}

\author{
HOUDE HAN ${ }^{\dagger}$ AND ZHIWEN ZHANG
}

\begin{abstract}
We develop a multiscale tailored finite point method (MsTFPM) for second order elliptic equations with rough or highly oscillatory coefficients. The finite point method has been tailored to some particular properties of the problem, so that it can capture the multiscale solutions using coarse meshes without resolving the fine scale structure of the solution. Several numerical examples in one- and two-dimensions are provided to show the accuracy and convergence of the proposed method. In addition, some analysis results based on the maximum principle for the onedimensional problem are proved.
\end{abstract}

Key words. Tailored finite point method (TFPM), multiple scales, maximum principle, rough coefficients, elliptic. equations.

AMS subject classifications. 35J25, 65N99, 65Y99.

\section{Introduction}

The second order elliptic boundary value problems with rough or highly oscillatory coefficients arise in many fields, such as composite materials and porous media $[1,2,3]$. In this paper, we consider the multiscale tailored finite point method (MsTFPM) for the second order elliptic boundary value problem with highly oscillatory coefficients given by

$$
\begin{aligned}
& -\nabla \cdot\left(A^{\epsilon}(\mathbf{x}) \nabla u^{\epsilon}(\mathbf{x})\right)+b^{\epsilon}(\mathbf{x}) u^{\epsilon}(\mathbf{x})=f^{\epsilon}(\mathbf{x}), \quad \mathbf{x} \in \Omega \\
& u^{\epsilon}(\mathbf{x})=0, \quad \mathbf{x} \in \partial \Omega .
\end{aligned}
$$

Here $\mathbf{x}=x \in R^{1}$ for the one-dimensional problem and $\mathbf{x}=(x, y) \in R^{2}$ for the twodimensional problem. $\Omega$ is a bounded domain, which is an interval for the onedimensional problem and a rectangle for the two-dimensional problem. The function $b^{\epsilon}(\mathbf{x}) \geq 0, \forall \mathbf{x} \in \bar{\Omega}$. The matrix function $A^{\epsilon}(\mathbf{x})$, function $b^{\epsilon}(\mathbf{x})$, and the force term $f^{\epsilon}(\mathbf{x})$ contain a small scale parameter $\epsilon$.

In practical applications, Equation (1.1) is the equation of steady state heat conduction through a composite material, with $u^{\epsilon}(\mathbf{x})$ and $A^{\epsilon}(\mathbf{x})$ interpreted as the temperature and the thermal conductivity. The Equation (1.1) is also the pressure equation in modelling two phase flow in porous media, with $u^{\epsilon}(\mathbf{x})$ and $A^{\epsilon}(\mathbf{x})$ interpreted as the pressure and the relative permeability tensor; see [8].

In the one dimensional case, $A^{\epsilon}(\mathbf{x})=a^{\epsilon}(x)$ and the Equation (1.1) becomes

$$
-\left(a^{\epsilon}(x) u^{\epsilon}(x)^{\prime}\right)^{\prime}+b^{\epsilon}(x) u^{\epsilon}(x)=f^{\epsilon}(x), \quad x \in \Omega
$$

In the two dimensional case, $A^{\epsilon}(\mathbf{x})$ is a $2 \times 2$ matrix function, defined by

$$
A^{\epsilon}(\mathbf{x})=\left(a_{i j}^{\epsilon}(\mathbf{x})\right)_{2 \times 2}, \quad \mathbf{x}=(x, y) \in \Omega .
$$

${ }^{*}$ Received: June 28, 2011; accepted (in revised version): November 30, 2011. Communicated by Pingwen Zhang.

${ }^{\dagger}$ Department of Mathematical Sciences, Tsinghua University, Beijing 100084, P.R. China (hhan@math.tsinghua.edu.cn).

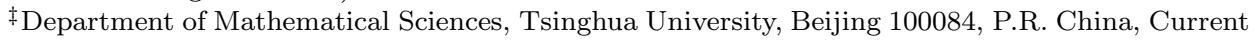
address: Applied Mathematics, Caltech, Pasadena, CA 91125, USA (zhangzw@caltech.edu). 
We assume the matrix $A^{\epsilon}(\mathbf{x})$ is positive definite with upper and lower bounds, namely, that there exist positive constants $m$ and $M$ such that

$$
m\|\xi\|^{2} \leq \sum_{i, j=1}^{2} a_{i j}^{\epsilon}(\mathbf{x}) \xi_{i} \xi_{j} \leq M\|\xi\|^{2}, \quad \xi=\left(\xi_{1}, \xi_{2}\right)^{T} \in R^{2}, \quad \mathbf{x}=(x, y) \in \bar{\Omega} .
$$

In the typical situation of multiscale modelling, one assumes that $A^{\epsilon}(\mathbf{x})=$ $\left(a_{i j}(\mathbf{x}, \epsilon)\right)_{2 \times 2}, b^{\epsilon}(\mathbf{x})=b(\mathbf{x}, \epsilon)$, and $f^{\epsilon}(\mathbf{x})=f(\mathbf{x}, \epsilon)$ are oscillatory functions involving a small scale parameter $\epsilon$. The solution oscillates rapidly and requires a very refined mesh to resolve. It is numerically difficult for traditional numerical methods to handle due to the tremendous amount of computer memory and CPU time.

There are a lot of studies on the numerical solutions to the problem (1.1)-(1.2). For instance, Babuska et al. proposed a generalized finite element methods approach to this kind of problem. In [4], theoretical proofs were provided for the one dimensional case and an arbitrary order approximation was obtained based on the continuous finite element method. The two dimensional case was considered in [5], where secondorder accurate elements were considered based on the non-conforming finite element methods. Engquist and Luo studied the convergence of the multigrid method for highly oscillatory elliptic problems on a new coarse-grid finite difference scheme; see $[6,7]$. Hou et al. developed the multiscale finite element methods (MsFEM) to study the multiscale problem. The main idea of the multiscale finite element methods is to construct multiscale bases which are adapted to the local properties of the differential operators within each element. The small scale information is then brought to the large scales through the coupling of the global stiffness matrix. Thus, the effect of small scales on the large scales is correctly captured; see $[8,9,10,11]$. In [12], E and Engquist proposed the heterogeneous multiscale method (HMM) for multiscale problems. The heterogeneous multiscale method consists of two main components: a macroscopic scheme for the macro-scale variables on a macro-scale grid and estimating the missing macroscopic data from the microscopic model. In [13], E et al. presented a systematic review of the heterogeneous multiscale method, including the fundamental designing philosophy and the error analysis. Recently, Shu et al. have developed a multiscale discontinuous Galerkin (DG) method for solving a class of second order elliptic problems with rough coefficients. They adopted a non-polynomial multiscale approximation space in the DG method to capture the multiscale solutions using coarse meshes without resolving the fine scale structure of the solution; see [15]. The multiscale finite element method and multiscale discontinuous Galerkin method are based on the finite element framework. After obtaining the local bases, one needs numerical integration to compute the global stiffness matrix and load vector.

The tailored finite point method (TFPM) is a new discrete method for solving differential equations numerically; see [16]. For each given problem, the discrete scheme has been tailored to some particular properties of the given problem. There are some successful applications of the tailored finite point method for solving singular perturbation problems [16, 17], interface problems [18], high frequency waves [19], and steady-state reaction-diffusion problems [20]. In this paper, we propose a multiscale tailored finite point method to deal with the second order elliptic problem (1.1)-(1.2) both in one- and two-dimensions.

The paper is organized as follows. In Section 2, we propose the multiscale tailored finite point method (MsTFPM) in one-dimension. In addition, we give some analysis results about the stability and convergence of the proposed method based on the maximum principle. We also extend our method to two-dimensional problems in 
Section 3. In Section 4, we use some numerical examples to show the efficiency of our new method. Finally in Section 5, we give some concluding remarks.

\section{MsTFPM in one-dimensional domains}

We study the second order elliptic equations with rough or highly oscillatory coefficients on the domain $\Omega=[0,1]$ :

$$
\begin{aligned}
& -\left(a^{\epsilon}(x) u^{\epsilon}(x)^{\prime}\right)^{\prime}+b^{\epsilon}(x) u^{\epsilon}(x)=f^{\epsilon}(x), \quad x \in(0,1) \\
& u^{\epsilon}(0)=0, \quad u^{\epsilon}(1)=0
\end{aligned}
$$

where $a^{\epsilon}(x)=a(x, \epsilon), b^{\epsilon}(x)=b(x, \epsilon)$, and $f^{\epsilon}(x)=f(x, \epsilon)$ are assumed to be oscillatory functions involving a small scale parameter $\epsilon$, or discontinuous functions. Moreover, we assume the coefficient $a^{\epsilon}(x)$ satisfies $0<\alpha \leq a^{\epsilon}(x) \leq \beta$, where $\alpha$ and $\beta$ are constants and $b^{\epsilon}(x) \geq 0$ for any $x \in[0,1]$. Let

$$
F=\max _{0 \leq x \leq 1}\left|f^{\epsilon}(x)\right|,
$$

which is independent of $\epsilon$.

2.1. Uniform estimate of the solution. We now give the uniform estimate of the solution $u^{\epsilon}(x)$ of the problem (2.1)-(2.2).

THEOREM 2.1.1. The solution $u^{\epsilon}(x)$ of the problem (2.1)-(2.2) is uniformly bounded on $\Omega$, namely,

$$
\max _{x \in \Omega}\left|u^{\epsilon}(x)\right| \leq C \max _{x \in \Omega}\left|f^{\epsilon}(x)\right|=C F .
$$

The constant $C$ depends only on the length of the domain $\Omega$ and $\alpha$, the lower bound of $a^{\epsilon}(x)$.

Proof. We define a differential operator $L^{\epsilon}$ on the solution $u^{\epsilon}(x)$ of Equation (2.1), that is

$$
\mathrm{L}^{\epsilon}\left(u^{\epsilon}(x)\right):=-\left(a^{\epsilon}(x) u^{\epsilon}(x)^{\prime}\right)^{\prime}+b^{\epsilon}(x) u^{\epsilon}(x)=f^{\epsilon}(x), \quad x \in(0,1) .
$$

To obtain a bound on the solution $u^{\epsilon}(x)$, we first define a comparison function

$$
w^{\epsilon}(x)=F \int_{0}^{x} \frac{1-s}{a^{\epsilon}(s)} d s .
$$

Obviously $w^{\epsilon}(x) \geq 0, \forall x \in[0,1]$. By a direct calculation, we obtain

$$
\mathrm{L}^{\epsilon}\left(w^{\epsilon}(x)\right) \geq F, \quad x \in(0,1) .
$$

If we write

$$
M^{\epsilon}(x)=w^{\epsilon}(x)+u^{\epsilon}(x), \quad x \in(0,1),
$$

then we have

$$
\mathrm{L}^{\epsilon}\left(M^{\epsilon}(x)\right) \geq F+f^{\epsilon}(x) \geq 0, \quad x \in(0,1) .
$$

According to the maximum principle [21], we find that $M^{\epsilon}(x)$ cannot be smaller than all the boundary values. Recall that

$$
\begin{aligned}
& M^{\epsilon}(0)=w^{\epsilon}(0)+u^{\epsilon}(0)=0, \\
& M^{\epsilon}(1)=w^{\epsilon}(1)+u^{\epsilon}(1) \geq 0,
\end{aligned}
$$


therefore we have the result $M^{\epsilon}(x) \geq 0, x \in[0,1]$, that is,

$$
u^{\epsilon}(x) \geq-F \int_{0}^{x} \frac{1-s}{a^{\epsilon}(s)} d s, \quad x \in[0,1] .
$$

Notice that this is a one-side bound. Similarly we define $M^{\epsilon}(x)=w^{\epsilon}(x)-u^{\epsilon}(x)$, and we get

$$
u^{\epsilon}(x) \leq F \int_{0}^{x} \frac{1-s}{a^{\epsilon}(s)} d s, \quad x \in[0,1] .
$$

Combining inequalities (2.5)-(2.6) together, we arrive at

$$
\left|u^{\epsilon}(x)\right| \leq F \int_{0}^{x} \frac{1-s}{a^{\epsilon}(s)} d s \leq \frac{F}{\alpha} \int_{0}^{x}(1-s) d s=\frac{F}{2 \alpha}:=C F, \quad x \in[0,1] .
$$

From (2.7), we immediately get that $\max _{x \in \Omega}\left|u^{\epsilon}(x)\right| \leq C \max _{x \in \Omega}\left|f^{\epsilon}(x)\right|$. The constant $\mathrm{C}$ depends only on the length of the domain $\Omega$ and $\alpha$, the lower bound of $a^{\epsilon}(x)$. Theorem 2.1 is proved completely.

The Theorem 2.1 shows that the upper bound of the absolute value of $u^{\epsilon}(x)$ does not depend on the small parameter $\epsilon$. We next show that similar result also holds in the numerical solution to the problem (2.1)-(2.2). Let $h=1 / K$ denote the mesh size, where $K$ is a positive integer. Then the mesh grid is given by

$$
\Omega_{h}=\left\{x_{j} \mid x_{j}=j h, \quad j=0, \cdots K\right\} .
$$

Suppose $U_{h}=\left\{U_{j} \mid 0 \leq j \leq K\right\}$ is a grid function on the grid $\Omega_{h}$. We discretize (2.1) by the finite difference scheme

$$
\left\{\begin{array}{l}
\frac{a_{j-\frac{1}{2}}^{\epsilon}\left(U_{j}-U_{j-1}\right)}{h^{2}}-\frac{a_{j+\frac{1}{2}}^{\epsilon}\left(U_{j+1}-U_{j}\right)}{h^{2}}+b_{j}^{\epsilon} U_{j}=f_{j}^{\epsilon}, \quad j=1, \cdots K-1, \\
U_{0}=U_{K}=0,
\end{array}\right.
$$

where $a_{j-\frac{1}{2}}^{\epsilon}=a^{\epsilon}\left(x_{j}-\frac{h}{2}\right), a_{j+\frac{1}{2}}^{\epsilon}=a^{\epsilon}\left(x_{j}+\frac{h}{2}\right), b_{j}^{\epsilon}=b^{\epsilon}\left(x_{j}\right)$ and $f_{j}^{\epsilon}=f^{\epsilon}\left(x_{j}\right)$.

ThEOREM 2.1.2. The numerical solution $\left\{U_{j}, j=0, \cdots K\right\}$ of the difference scheme (2.8) satisfies the estimate

$$
\max _{j=0, \cdots K}\left|U_{j}\right| \leq C \max _{j=0, \cdots K}\left|f_{j}\right| .
$$

The constant $C$ depends only on the length of the domain $\Omega$ and $\alpha$, the lower bound of $a^{\epsilon}(x)$.

Proof. We now define an operator $\mathrm{L}_{h}^{\epsilon}$ on the grid function $U_{h}$,

$$
\mathrm{L}_{h}^{\epsilon} U_{j}:=\frac{a_{j-\frac{1}{2}}^{\epsilon}\left(U_{j}-U_{j-1}\right)}{h^{2}}-\frac{a_{j+\frac{1}{2}}^{\epsilon}\left(U_{j+1}-U_{j}\right)}{h^{2}}+b_{j}^{\epsilon} U_{j}=f_{j}^{\epsilon}, \quad j=1, \cdots K-1 .
$$

To obtain a bound for the grid function $U_{h}$, we define a comparison function on the grid $\Omega_{h}$

$$
\left\{\begin{array}{l}
W_{0}=0 \\
W_{j}=F \sum_{k=1}^{j} \frac{\left(1-x_{k-\frac{1}{2}}\right)}{a_{k-\frac{1}{2}}^{\epsilon}} h, \quad j=1, \cdots, K
\end{array}\right.
$$


By a direct calculation, we obtain

$$
\mathrm{L}_{h}^{\epsilon} W_{j} \geq F, \quad j=1, \cdots K-1 .
$$

If we define $M_{j}=W_{j}+U_{j}, j=1, \cdots K-1$, then we get $\mathrm{L}_{h}^{\epsilon} M_{j} \geq F+f_{j} \geq 0, j=1, \cdots K-$ 1. According to the discrete maximum principle [22], $M_{j}$ cannot be smaller than all the boundary values. Recall that $M_{0}=W_{0}+U_{0}=0$ and $M_{K}=W_{K}+U_{K} \geq 0$, therefore we have the result $M_{j} \geq 0, j=0, \cdots K$, namely,

$$
U_{j} \geq-F \sum_{k=1}^{j} \frac{\left(1-x_{k-\frac{1}{2}}\right)}{a_{k-\frac{1}{2}}^{\epsilon}} h, \quad j=0, \cdots K .
$$

Next we define $M_{j}=W_{j}-U_{j}, j=1, \cdots K-1$ and repeat the same analysis, then we get

$$
U_{j} \leq F \sum_{k=1}^{j} \frac{\left(1-x_{k-\frac{1}{2}}\right)}{a_{k-\frac{1}{2}}^{\epsilon}} h, \quad j=0, \cdots K .
$$

Combining inequalities (2.12)-(2.13) together, we arrive at

$$
\left|U_{j}\right| \leq F \sum_{k=1}^{j} \frac{\left(1-x_{k-\frac{1}{2}}\right)}{a_{k-\frac{1}{2}}^{\epsilon}} h, \quad j=0, \cdots K .
$$

From (2.14), we immediately obtain

$$
\max _{j=0, \cdots K}\left|U_{j}\right| \leq F \sum_{k=1}^{K} \frac{\left(1-x_{k-\frac{1}{2}}\right)}{a_{k-\frac{1}{2}}^{\epsilon}} h \leq \frac{F}{2 \alpha}:=C F .
$$

The constant $\mathrm{C}$ depends only on the length of the domain $\Omega$ and $\alpha$, the lower bound of $a^{\epsilon}(x)$. Theorem 2.2 is proved completely.

Let $e_{j}=U_{j}-u^{\epsilon}\left(x_{j}\right), j=1, \cdots K-1$, then

$$
\mathrm{L}_{h}^{\epsilon} e_{j}=-\frac{1}{12} a_{j}^{\epsilon} \frac{d^{4} u^{\epsilon}\left(x_{j}\right)}{d x^{4}} h^{2}-\frac{1}{6} \frac{d a^{\epsilon}\left(x_{j}\right)}{d x} \frac{d^{3} u^{\epsilon}\left(x_{j}\right)}{d x^{3}} h^{2}+R^{\epsilon}\left(x_{j}, h\right):=T_{j}^{\epsilon} h^{2},
$$

where $R^{\epsilon}\left(x_{j}, h\right)$ is the remainder term involving high-order derivatives. We have the estimate $\left|R^{\epsilon}\left(x_{j}, h\right)\right| \leq C^{\epsilon} h^{3}$. By the Theorem 2.1.2 and (2.16), we immediately get

THEOREM 2.1.3. In the difference scheme (2.8), the following error estimate holds:

$$
\max _{j=1, \cdots K-1}\left|e_{j}\right| \leq C h^{2} \max _{j=1, \cdots K-1}\left|T_{j}^{\epsilon}\right|
$$

where $C$ depends only on the length of the domain $\Omega$ and $\alpha$, the lower bound of $a^{\epsilon}(x)$.

2.2. Numerical scheme $I$. We consider the numerical solution to the problem (2.1)-(2.2) by the multiscale tailored finite point method. Let $H=1 / N$ denote the coarse mesh size, where $N$ is a positive integer. Then the coarse grid is given by

$$
\Omega_{H}=\left\{x_{i} \mid x_{i}=i H, \quad i=0, \cdots N\right\} .
$$


Suppose $U=\left\{u_{i} \mid 0 \leq i \leq N\right\}$ is a grid function on the coarse grid $\Omega_{H}$. We propose the first multiscale tailored finite point scheme to obtain the numerical solution of the problem (2.1)-(2.2).

For the interior gird points $\left\{x_{i} \mid i=1, \cdots N-1\right\}$, we consider the cell $I_{i}=$ $\left[x_{i-1}, x_{i+1}\right]$ and $x_{i}$ is the center of the $I_{i}$. On each cell $I_{i}$, we consider the following three local problems:

$$
\begin{aligned}
& \left\{\begin{array}{l}
-\left(a^{\epsilon}(x) p_{i}^{-}(x)^{\prime}\right)^{\prime}+b^{\epsilon}(x) p_{i}^{-}(x)=0, \quad x \in\left(x_{i-1}, x_{i+1}\right), \\
p_{i}^{-}\left(x_{i-1}\right)=1, \quad p_{i}^{-}\left(x_{i+1}\right)=0,
\end{array}\right. \\
& \left\{\begin{array}{l}
-\left(a^{\epsilon}(x) p_{i}^{+}(x)^{\prime}\right)^{\prime}+b^{\epsilon}(x) p_{i}^{+}(x)=0, \quad x \in\left(x_{i-1}, x_{i+1}\right), \\
p_{i}^{+}\left(x_{i-1}\right)=0, \quad p_{i}^{+}\left(x_{i+1}\right)=1,
\end{array}\right. \\
& \left\{\begin{array}{l}
-\left(a^{\epsilon}(x) p_{i}^{f}(x)^{\prime}\right)^{\prime}+b^{\epsilon}(x) p_{i}^{f}(x)=f^{\epsilon}(x), \quad x \in\left(x_{i-1}, x_{i+1}\right), \\
p_{i}^{f}\left(x_{i-1}\right)=0, \quad p_{i}^{f}\left(x_{i+1}\right)=0 .
\end{array}\right.
\end{aligned}
$$

The local problems (2.18)-(2.20) have unique solutions separately. Furthermore we can see that on each cell $I_{i}$, the solution $u^{\epsilon}(x)$ of the problem (2.1)-(2.2) can be represented by

$$
u^{\epsilon}(x)=u^{\epsilon}\left(x_{i-1}\right) p_{i}^{-}(x)+u^{\epsilon}\left(x_{i+1}\right) p_{i}^{+}(x)+p_{i}^{f}(x), \quad \forall x \in\left[x_{i-1}, x_{i+1}\right] .
$$

Letting $x=x_{i}$ in the Equation (2.21) we arrive at

$$
u^{\epsilon}\left(x_{i}\right)=u^{\epsilon}\left(x_{i-1}\right) p_{i}^{-}\left(x_{i}\right)+u^{\epsilon}\left(x_{i+1}\right) p_{i}^{+}\left(x_{i}\right)+p_{i}^{f}\left(x_{i}\right) .
$$

Recall that $u_{i}$ represents the value of the solution of $u^{\epsilon}(x)$ on the grid point $x=x_{i}$, namely $u_{i}=u^{\epsilon}\left(x_{i}\right)$. If we can obtain all the exact solutions of the local problems (2.18)-(2.20) for $i=1,2, \cdots, N-1$, then from (2.22) we immediately obtain the following discrete scheme for the problem (2.1)-(2.2) on the coarse grid $\Omega_{H}$ :

$$
\left\{\begin{array}{l}
u_{i}=\alpha_{i} u_{i-1}+\beta_{i} u_{i+1}+\gamma_{i}, \quad i=1, \cdots, N-1 \\
u_{0}=0, \quad u_{N}=0
\end{array}\right.
$$

with $\alpha_{i}=p_{i}^{-}\left(x_{i}\right), \beta_{i}=p_{i}^{+}\left(x_{i}\right)$, and $\gamma_{i}=p_{i}^{f}\left(x_{i}\right), i=1, \cdots, N-1$.

LEMMA 2.2.1. In the discrete scheme (2.23) for the multiscale elliptic problem (2.1)-(2.2), the constants $\left\{\alpha_{i}, \beta_{i}, i=1, \cdots, N-1\right\}$ satisfy the following conditions:

$$
\begin{aligned}
& 0<\alpha_{i}<1, \\
& 0<\beta_{i}<1, \\
& 0<\alpha_{i}+\beta_{i} \leq 1,
\end{aligned}
$$

where $i=1, \cdots, N-1$.

Proof. Since $\alpha_{i}=p_{i}^{-}\left(x_{i}\right)$ and $p_{i}^{-}(x)$ is the solution of the local cell problem (2.18) on the cell $I_{i}=\left[x_{i-1}, x_{i+1}\right]$. By the maximum principle [21], we find that

$$
0 \leq p_{i}^{-}(x) \leq 1, \quad \forall x \in\left[x_{i-1}, x_{i+1}\right]
$$

and

$$
0<\alpha_{i}=p_{i}^{-}\left(x_{i}\right)<1, \quad i=1, \cdots, N-1
$$


Namely the inequality $0<\alpha_{i}<1$ holds. The proof of the inequality $0<\beta_{i}<1$ is similar. Furthermore, let $p_{i}(x)=p_{i}^{-}(x)+p_{i}^{+}(x)$. Then $p_{i}(x)$ satisfies

$$
\left\{\begin{array}{l}
-\left(a^{\epsilon}(x) p(x)^{\prime}\right)^{\prime}+b^{\epsilon}(x) p(x)=0, \quad x \in\left(x_{i-1}, x_{i+1}\right), \\
p\left(x_{i-1}\right)=1, \quad p\left(x_{i+1}\right)=1 .
\end{array}\right.
$$

By the maximum principle [21], we can see that

$$
0<p_{i}(x) \leq 1, \quad \forall x \in\left[x_{i-1}, x_{i+1}\right] .
$$

Since $\alpha_{i}+\beta_{i}=p_{i}\left(x_{i}\right)$, the inequality $0<\alpha_{i}+\beta_{i} \leq 1$ follows immediately.

The system of equations in the scheme (2.23) is tridiagonal, so the matrix of the system is a diagonal matrix. The Lemma 2.2.1 ensures that the matrix is diagonal dominant, thus it is invertible. We immediately obtain

TheOREM 2.2.2. The discrete scheme (2.23) for the multiscale elliptic problem (2.1)-(2.2) has a unique solution $\left\{u_{i} \mid u_{i}=u^{\epsilon}\left(x_{i}\right), 0 \leq i \leq N\right\}$.

The Theorem 2.2.2 means that from the discrete scheme (2.23) we can get the exact solution of the problem (2.1)-(2.2) on the coarse grid points $x=x_{i}, 0 \leq i \leq N$, if we can solve the local problems (2.18)-(2.20) for $i=1,2, \cdots, N-1$ exactly. In this case, the discrete scheme (2.23) is the numerical scheme for the problem (2.1)-(2.2). This is the essence of the tailored finite point method [16, 17, 18, 19, 20]. In general, however, one cannot obtain the exact solution of the local problems (2.18)-(2.20) for $i=1, \cdots, N-1$. Therefore the constants $\alpha_{i}, \beta_{i}, \gamma_{i}, i=1, \cdots, N-1$ are unknown and one cannot use the discrete scheme $(2.23)$ to obtain the numerical solution of the problem (2.1)-(2.2) on the coarse grid $\Omega_{H}$.

In practical computation, one can first solve the local problems (2.18)-(2.20) numerically on each cell to obtain the constants $\alpha_{i}, \beta_{i}, \gamma_{i}, i=1, \cdots, N-1$ approximately, then use the discrete scheme (2.23) with $\alpha_{i}, \beta_{i}, \gamma_{i}$ replaced by their approximations to obtain the numerical solution of the problem (2.1)-(2.2). This is the main idea of the multiscale tailored finite point method proposed in this paper.

Here we solve the local cell problems (2.18)-(2.20) numerically to demonstrate this idea. Let $h=H / M$ denote the fine mesh size, where $H=1 / N$ is the pre-defined coarse mesh size and $M$ is a positive integer. On the cell $I_{i}, i=1, \cdots, N-1$, we define a fine mesh grid by

$$
\Omega_{i, h}=\left\{x_{i, j} \mid x_{i, j}=x_{i}+j h, \quad j=-M, \cdots, 0, \cdots, M\right\},
$$

with $x_{i,-M}=x_{i-1}, x_{i, 0}=x_{i}, x_{i, M}=x_{i+1}$. In fact, all of the local fine mesh grids $\Omega_{i, h}$ form a fine mesh grid on $[0,1]$,

$$
\Omega_{h}=\left\{y_{j} \mid y_{j}=j h, \quad j=0,1, \cdots, N M\right\} .
$$

We solve the local cell problems (2.18)-(2.20) numerically on each local fine mesh grid $\Omega_{i, h}$. Let $p_{i, h}^{-}(x), p_{i, h}^{+}(x)$, and $p_{i, h}^{f}(x), x \in \Omega_{i, h}, i=1, \cdots, N-1$ be the numerical solutions, respectively. From the proof of the Lemma 2.2.1, we know that

$$
\begin{array}{r}
0<p_{i, h}^{-}(x), \quad p_{i, h}^{+}(x)<1, \quad x \in \Omega_{i, h}, \quad i=1, \cdots, N-1, \\
0<p_{i, h}^{-}(x)+p_{i, h}^{+}(x) \leq 1, \quad x \in \Omega_{i, h}, \quad i=1, \cdots, N-1 .
\end{array}
$$


Using the numerical solutions $p_{i, h}^{-}(x), p_{i, h}^{+}(x)$, and $p_{i, h}^{f}(x), x \in \Omega_{i, h}, i=1, \cdots, N-1$, we construct the numerical solutions on each local fine mesh grid $\Omega_{i, h}$

$$
u_{h}^{\epsilon}(x)=u_{h}^{\epsilon}\left(x_{i-1}\right) p_{i, h}^{-}(x)+u_{h}^{\epsilon}\left(x_{i+1}\right) p_{i, h}^{+}(x)+p_{i, h}^{f}(x), \quad x \in \Omega_{i, h}, \quad i=1, \cdots, N-1 .
$$

Letting $x=x_{i, 0}=x_{i}$, we arrive at

$$
u_{h}^{\epsilon}\left(x_{i}\right)=u_{h}^{\epsilon}\left(x_{i-1}\right) p_{i, h}^{-}\left(x_{i}\right)+u_{h}^{\epsilon}\left(x_{i+1}\right) p_{i, h}^{+}\left(x_{i}\right)+p_{i, h}^{f}\left(x_{i}\right), \quad i=1, \cdots, N-1 .
$$

We obtain a multiscale tailored finite point scheme for the multiscale elliptic problem (2.1)-(2.2)

$$
\left\{\begin{array}{l}
u_{i, h}=\alpha_{i, h} u_{i-1, h}+\beta_{i, h} u_{i+1, h}+\gamma_{i, h}, \quad i=1, \cdots, N-1, \\
u_{0, h}=0, \quad u_{N, h}=0,
\end{array}\right.
$$

with $\alpha_{i, h}=p_{i, h}^{-}\left(x_{i}\right), \beta_{i, h}=p_{i, h}^{+}\left(x_{i}\right)$, and $\gamma_{i, h}=p_{i, h}^{f}\left(x_{i}\right), i=1, \cdots, N-1$. From the properties of the local numerical solutions, we immediately find.

Lemma 2.2.3. In the multiscale tailored finite point scheme (2.28) for the multiscale elliptic problem (2.1)-(2.2), we have the following estimates:

$$
\begin{aligned}
& 0<\alpha_{i, h}<1, \\
& 0<\beta_{i, h}<1, \\
& 0<\alpha_{i, h}+\beta_{i, h} \leq 1, \quad i=1, \cdots, N-1 .
\end{aligned}
$$

The system of equations in the scheme (2.28) is tridiagonal. The Lemma 2.2.3 ensures that the matrix is diagonal dominant, thus it is invertible. We immediately obtain

THEOREM 2.2.4. The multiscale tailored finite point scheme (2.28) for the multiscale elliptic problem (2.1)-(2.2) has a unique numerical solution $\left\{u_{i, h} \mid 0 \leq i \leq N\right\}$.

2.3. Numerical scheme II. We propose the second multiscale tailored finite point scheme for the given problem (2.1)-(2.2) based on the flux continuity condition, which can specially be applied to problems with discontinuous coefficient. For the interior grid points $\left\{x_{i} \mid i=1, \cdots N-1\right\}$, we consider the left cell $I_{i}^{L}=\left[x_{i-1}, x_{i}\right]$ and the right cell $I_{i}^{R}=\left[x_{i}, x_{i+1}\right]$. On the left cell $I_{i}^{L}$, we consider the following three local cell problems:

$$
\begin{aligned}
& \left\{\begin{array}{l}
-\left(a^{\epsilon}(x) p_{i-\frac{1}{2}}^{+}(x)^{\prime}\right)^{\prime}+b^{\epsilon}(x) p_{i-\frac{1}{2}}^{+}(x)=0, \quad x \in\left(x_{i-1}, x_{i}\right), \\
p_{i-\frac{1}{2}}^{+}\left(x_{i-1}\right)=0, \quad p_{i-\frac{1}{2}}^{+}\left(x_{i}\right)=1,
\end{array}\right. \\
& \left\{\begin{array}{l}
-\left(a^{\epsilon}(x) p_{i-\frac{1}{2}}^{-}(x)^{\prime}\right)^{\prime}+b^{\epsilon}(x) p_{i-\frac{1}{2}}^{-}(x)=0, \quad x \in\left(x_{i-1}, x_{i}\right), \\
p_{i-\frac{1}{2}}^{-}\left(x_{i-1}\right)=1, \quad p_{i-\frac{1}{2}}^{-}\left(x_{i}\right)=0,
\end{array}\right. \\
& \left\{\begin{array}{l}
-\left(a^{\epsilon}(x) p_{i-\frac{1}{2}}^{f}(x)^{\prime}\right)^{\prime}+b^{\epsilon}(x) p_{i-\frac{1}{2}}^{f}(x)=f^{\epsilon}(x), \quad x \in\left(x_{i-1}, x_{i}\right), \\
p_{i-\frac{1}{2}}^{f}\left(x_{i-1}\right)=0, \quad p_{i-\frac{1}{2}}^{f}\left(x_{i}\right)=0 .
\end{array}\right.
\end{aligned}
$$

On the right cell $I_{i}^{R}$, we consider the following three local cell problems:

$$
\left\{\begin{array}{l}
-\left(a^{\epsilon}(x) p_{i+\frac{1}{2}}^{+}(x)^{\prime}\right)^{\prime}+b^{\epsilon}(x) p_{i+\frac{1}{2}}^{+}(x)=0, \quad x \in\left(x_{i}, x_{i+1}\right), \\
p_{i+\frac{1}{2}}^{+}\left(x_{i}\right)=0, \quad p_{i+\frac{1}{2}}^{+}\left(x_{i+1}\right)=1
\end{array}\right.
$$




$$
\begin{gathered}
\left\{\begin{array}{l}
-\left(a^{\epsilon}(x) p_{i+\frac{1}{2}}^{-}(x)^{\prime}\right)^{\prime}+b^{\epsilon}(x) p_{i+\frac{1}{2}}^{-}(x)=0, \quad x \in\left(x_{i}, x_{i+1}\right), \\
p_{i+\frac{1}{2}}^{-}\left(x_{i}\right)=1, \quad p_{i+\frac{1}{2}}^{-}\left(x_{i+1}\right)=0,
\end{array}\right. \\
\left\{\begin{array}{l}
-\left(a^{\epsilon}(x) p_{i+\frac{1}{2}}^{f}(x)^{\prime}\right)^{\prime}+b^{\epsilon}(x) p_{i+\frac{1}{2}}^{f}(x)=f^{\epsilon}(x), \quad x \in\left(x_{i}, x_{i+1}\right), \\
p_{i+\frac{1}{2}}^{f}\left(x_{i}\right)=0, \quad p_{i+\frac{1}{2}}^{f}\left(x_{i+1}\right)=0 .
\end{array}\right.
\end{gathered}
$$

The local problems (2.29)-(2.31) and (2.32)-(2.34) have unique solutions separately. Furthermore we can see that on the left cell $I_{i}^{L}$, the solution $u^{\epsilon}(x)$ of the problem (2.1)-(2.2) can be represented by

$$
u^{\epsilon}(x)=u^{\epsilon}\left(x_{i-1}\right) p_{i-\frac{1}{2}}^{-}(x)+u^{\epsilon}\left(x_{i}\right) p_{i-\frac{1}{2}}^{+}(x)+p_{i-\frac{1}{2}}^{f}(x), \quad \forall x \in\left[x_{i-1}, x_{i}\right] .
$$

On the right cell $I_{i}^{R}$, the solution $u^{\epsilon}(x)$ of the problem (2.1)-(2.2) can be represented by

$$
u^{\epsilon}(x)=u^{\epsilon}\left(x_{i}\right) p_{i+\frac{1}{2}}^{-}(x)+u^{\epsilon}\left(x_{i+1}\right) p_{i+\frac{1}{2}}^{+}(x)+p_{i+\frac{1}{2}}^{f}(x), \quad \forall x \in\left[x_{i}, x_{i+1}\right] .
$$

On the point $x_{i}, u^{\epsilon}(x)$ satisfies the continuity condition

$$
\left.u^{\epsilon}(x)\right|_{x=x_{i}^{-}}=\left.u^{\epsilon}(x)\right|_{x=x_{i}^{+}}
$$

and the flux conservative relation

$$
\left.\left(a^{\epsilon}(x) u^{\epsilon}(x)^{\prime}\right)\right|_{x=x_{i}^{-}}=\left.\left(a^{\epsilon}(x) u^{\epsilon}(x)^{\prime}\right)\right|_{x=x_{i}^{+}}
$$

By the equations (2.35)-(2.38) we immediately arrive at

$$
\begin{aligned}
& a^{\epsilon}\left(x_{i}^{-}\right)\left[u^{\epsilon}\left(x_{i-1}\right)\left(p_{i-\frac{1}{2}}^{-}\right)^{\prime}\left(x_{i}^{-}\right)+u^{\epsilon}\left(x_{i}\right)\left(p_{i-\frac{1}{2}}^{+}\right)^{\prime}\left(x_{i}^{-}\right)+\left(p_{i-\frac{1}{2}}^{f}\right)^{\prime}\left(x_{i}^{-}\right)\right] \\
= & a^{\epsilon}\left(x_{i}^{+}\right)\left[u^{\epsilon}\left(x_{i}\right)\left(p_{i+\frac{1}{2}}^{-}\right)^{\prime}\left(x_{i}^{+}\right)+u^{\epsilon}\left(x_{i+1}\right)\left(p_{i+\frac{1}{2}}^{+}\right)^{\prime}\left(x_{i}^{+}\right)+\left(p_{i+\frac{1}{2}}^{f}\right)^{\prime}\left(x_{i}^{+}\right)\right],
\end{aligned}
$$

where $i=1, \cdots, N-1$. According to our assumption, we have $a^{\epsilon}\left(x_{i}^{-}\right)>0$ and $a^{\epsilon}\left(x_{i}^{+}\right)>$ 0 . By the strong maximum principle [21], also called the Hopf Lemma, we have $\left(p_{i-\frac{1}{2}}^{+}\right)^{\prime}\left(x_{i}^{-}\right)>0$ and $\left(p_{i+\frac{1}{2}}^{-}\right)^{\prime}\left(x_{i}^{+}\right)<0$. Therefore we get

$$
D_{i}^{\epsilon}:=a^{\epsilon}\left(x_{i}^{-}\right)\left(p_{i-\frac{1}{2}}^{+}\right)^{\prime}\left(x_{i}^{-}\right)-a^{\epsilon}\left(x_{i}^{+}\right)\left(p_{i+\frac{1}{2}}^{-}\right)^{\prime}\left(x_{i}^{+}\right)>0 .
$$

From (2.39), we obtain the following equations:

$$
\left\{\begin{array}{l}
u^{\epsilon}\left(x_{i}\right)=A_{i} u^{\epsilon}\left(x_{i-1}\right)+B_{i} u^{\epsilon}\left(x_{i+1}\right)+C_{i}, \quad i=1, \cdots, N-1, \\
u^{\epsilon}\left(x_{0}\right)=0, \quad u^{\epsilon}\left(x_{N}\right)=0,
\end{array}\right.
$$

where

$$
\begin{aligned}
& A_{i}=-a^{\epsilon}\left(x_{i}^{-}\right)\left(p_{i-\frac{1}{2}}^{-}\right)^{\prime}\left(x_{i}^{-}\right) / D_{i}^{\epsilon}, \\
& B_{i}=a^{\epsilon}\left(x_{i}^{+}\right)\left(p_{i+\frac{1}{2}}^{+}\right)^{\prime}\left(x_{i}^{+}\right) / D_{i}^{\epsilon}, \\
& C_{i}=\left(a^{\epsilon}\left(x_{i}^{+}\right)\left(p_{i+\frac{1}{2}}^{f}\right)^{\prime}\left(x_{i}^{+}\right)-a^{\epsilon}\left(x_{i}^{-}\right)\left(p_{i-\frac{1}{2}}^{f}\right)^{\prime}\left(x_{i}^{-}\right)\right) / D_{i}^{\epsilon} .
\end{aligned}
$$


Recall that $u_{i}$ represents the value of the solution $u^{\epsilon}(x)$ on the grid point $x=x_{i}$, namely, $u_{i}=u^{\epsilon}\left(x_{i}\right)$. We obtain the following discrete scheme for the problem (2.1)(2.2) on the coarse mesh $\Omega_{H}$ :

$$
\left\{\begin{array}{l}
u_{i}=A_{i} u_{i-1}+B_{i} u_{i+1}+C_{i}, \quad i=1, \cdots, N-1, \\
u_{0}=0, \quad u_{N}=0
\end{array}\right.
$$

where $A_{i}, B_{i}, C_{i}$ are defined in the Equation (2.42).

Lemma 2.3.1. In the discrete scheme (2.43) for the multiscale elliptic problem (2.1)-(2.2), we have the following estimates:

$$
\begin{aligned}
& 0<A_{i}<1, \\
& 0<B_{i}<1, \\
& 0<A_{i}+B_{i} \leq 1,
\end{aligned}
$$

where $i=1, \cdots, N-1$.

Proof. After solving the local cell problems (2.29)-(2.31) and (2.32)-(2.34), we have the solutions $p_{i-\frac{1}{2}}^{-}(x), p_{i-\frac{1}{2}}^{+}(x), p_{i-\frac{1}{2}}^{f}(x)$ on $\left[x_{i-1}, x_{i}\right]$ and $p_{i+\frac{1}{2}}^{-}(x), p_{i+\frac{1}{2}}^{+}(x)$, $p_{i+\frac{1}{2}}^{f}(x)$ on $\left[x_{i}, x_{i+1}\right]$, respectively. Then the solution $p_{i}^{-}(x)$ of the problem (2.18) can be written as

$$
p_{i}^{-}(x)=\left\{\begin{array}{l}
p_{i-\frac{1}{2}}^{-}(x)+\lambda_{i}^{1} p_{i-\frac{1}{2}}^{+}(x), \quad x \in\left[x_{i-1}, x_{i}\right], \\
\lambda_{i}^{2} p_{i+\frac{1}{2}}^{-}(x), \quad x \in\left[x_{i}, x_{i+1}\right],
\end{array}\right.
$$

where the constants $\lambda_{i}^{1}$ and $\lambda_{i}^{2}$ can be determined by the conditions

$$
\left\{\begin{array}{l}
p_{i}^{-}\left(x_{i}^{-}\right)=p_{i}^{-}\left(x_{i}^{+}\right), \\
a^{\epsilon}\left(x_{i}^{-}\right)\left(p_{i}^{-}\left(x_{i}^{-}\right)\right)^{\prime}=a^{\epsilon}\left(x_{i}^{+}\right)\left(p_{i}^{-}\left(x_{i}^{+}\right)\right)^{\prime} .
\end{array}\right.
$$

Plugging the definition of the solution $p_{i}^{-}(x)$ into the conditions (2.45), we obtain that

$$
\lambda_{i}^{1}=\lambda_{i}^{2}=A_{i}=\frac{-a^{\epsilon}\left(x_{i}^{-}\right)\left(p_{i-\frac{1}{2}}^{-}\right)^{\prime}\left(x_{i}^{-}\right)}{a^{\epsilon}\left(x_{i}^{-}\right)\left(p_{i-\frac{1}{2}}^{+}\right)^{\prime}\left(x_{i}^{-}\right)-a^{\epsilon}\left(x_{i}^{+}\right)\left(p_{i+\frac{1}{2}}^{-}\right)^{\prime}\left(x_{i}^{+}\right)} .
$$

Similarly the solution $p_{i}^{+}(x)$ of the problem (2.19) can be written as

$$
p_{i}^{+}(x)=\left\{\begin{array}{l}
\mu_{i}^{1} p_{i-\frac{1}{2}}^{+}(x), \quad x \in\left[x_{i-1}, x_{i}\right], \\
\mu_{i}^{2} p_{i+\frac{1}{2}}^{-}(x)+p_{i+\frac{1}{2}}^{+}(x), \quad x \in\left[x_{i}, x_{i+1}\right],
\end{array}\right.
$$

with

$$
\mu_{i}^{1}=\mu_{i}^{2}=B_{i}=\frac{a^{\epsilon}\left(x_{i}^{+}\right)\left(p_{i+\frac{1}{2}}^{+}\right)^{\prime}\left(x_{i}^{+}\right)}{a^{\epsilon}\left(x_{i}^{-}\right)\left(p_{i-\frac{1}{2}}^{+}\right)^{\prime}\left(x_{i}^{-}\right)-a^{\epsilon}\left(x_{i}^{+}\right)\left(p_{i+\frac{1}{2}}^{-}\right)^{\prime}\left(x_{i}^{+}\right)} .
$$

Finally, the solution $p_{i}^{f}(x)$ of the problem (2.20) can be written as

$$
p_{i}^{f}(x)= \begin{cases}p_{i-\frac{1}{2}}^{f}(x)+\nu_{i}^{1} p_{i-\frac{1}{2}}^{+}(x), & x \in\left[x_{i-1}, x_{i}\right], \\ p_{i+\frac{1}{2}}^{f}(x)+\nu_{i}^{2} p_{i+\frac{1}{2}}^{-}(x), & x \in\left[x_{i}, x_{i+1}\right],\end{cases}
$$


with

$$
\nu_{i}^{1}=\nu_{i}^{2}=C_{i}=\frac{a^{\epsilon}\left(x_{i}^{+}\right)\left(p_{i+\frac{1}{2}}^{f}\right)^{\prime}\left(x_{i}^{+}\right)-a^{\epsilon}\left(x_{i}^{-}\right)\left(p_{i-\frac{1}{2}}^{f}\right)^{\prime}\left(x_{i}^{-}\right)}{a^{\epsilon}\left(x_{i}^{-}\right)\left(p_{i-\frac{1}{2}}^{+}\right)^{\prime}\left(x_{i}^{-}\right)-a^{\epsilon}\left(x_{i}^{+}\right)\left(p_{i+\frac{1}{2}}^{-}\right)^{\prime}\left(x_{i}^{+}\right)} .
$$

Recalling the equations $(2.21),(2.22)$, and (2.23) in the numerical scheme I, we have the corresponding relations $A_{i}=\alpha_{i}, B_{i}=\beta_{i}$, and $C_{i}=\gamma_{i}, i=1, \cdots, N-1$. Then the results $0<A_{i}<1,0<B_{i}<1$, and $0<A_{i}+B_{i} \leq 1, i=1, \cdots, N-1$, are immediately obtained by Lemma 2.2.1.

In general one cannot obtain the exact solution of the local problems (2.29)-(2.31) and (2.32)-(2.34) for $i=1, \cdots, N-1$. Therefore one cannot use the discrete scheme (2.43) to obtain the numerical solution of the problem (2.1)-(2.2). The same as the numerical scheme I, one can first solve the local problems (2.29)-(2.31) and (2.32)(2.34) numerically on each cell to obtain the approximations of the constants $A_{i}, B_{i}$, $C_{i}, i=1, \cdots, N-1$, then use the discrete scheme (2.43) with $A_{i}, B_{i}, C_{i}$ replaced by their approximations to obtain the numerical solution of the problem (2.1)-(2.2). We omit the details here.

REMARK 2.3.2. By either numerical scheme (2.23) or (2.43) we can obtain the numerical solution of $u^{\epsilon}(x)$ on the coarse grid $\Omega_{H}$. We can also recover the numerical solution of $u^{\epsilon}(x)$ on the fine scale by using (2.21) or (2.35)-(2.36). In this case, we need to save all the numerical solutions of the local cell problems (2.18)-(2.20) or (2.29)-(2.34).

\section{MsTFPM in two-dimensional domain}

We now consider the second order elliptic equations with rough or highly oscillatory coefficients on a square domain $\Omega=[0,1] \times[0,1]$ :

$$
\begin{aligned}
& -\nabla \cdot\left(A^{\epsilon}(\mathbf{x}) \nabla u^{\epsilon}(\mathbf{x})\right)+b^{\epsilon}(\mathbf{x}) u^{\epsilon}(\mathbf{x})=f^{\epsilon}(\mathbf{x}), \quad \mathbf{x} \in \Omega, \\
& u^{\epsilon}(\mathbf{x})=0, \quad \mathbf{x} \in \partial \Omega,
\end{aligned}
$$

where $\mathbf{x}=(x, y) \in R^{2}$. The operator $A^{\epsilon}(\mathbf{x})$ is given by (1.4) and the coefficient $b^{\epsilon}(\mathbf{x}) \geq 0$. For the simplicity in the statement of the MsTFPM, we assume that $a_{12}^{\epsilon}(\mathbf{x})=a_{21}^{\epsilon}(\mathbf{x})=0$ in the $A^{\epsilon}(\mathbf{x})$. For general matrix functions $A^{\epsilon}(\mathbf{x})=\left(a_{i j}^{\epsilon}(\mathbf{x})\right)_{2 \times 2}$, the MsTFPM can be used directly without any difficulty.

Let $H=1 / N$ denote the coarse mesh size, where $N$ is a positive integer. We divide the domain $\Omega$ by a set of lines parallel to the $\mathrm{x}-, \mathrm{y}$-axis to form a coarse mesh grid. The crossing points set $\Omega_{H}$ is called the coarse grid

$$
\Omega_{H}=\left\{\left(x_{i}, y_{j}\right) \mid x_{i}=i H, \quad y_{j}=j H, \quad i=0, \ldots, N, \quad j=0, \ldots, N\right\} .
$$

Suppose $U=\left\{u_{i j} \mid 0 \leq i \leq N, 0 \leq j \leq N\right\}$ is a grid function defined on the coarse gird $\Omega_{H}$. We present two multiscale tailored finite point schemes to obtain the numerical solution of the problem (3.1)-(3.2).

3.1. Numerical scheme I. For the interior grid points $\left\{\left(x_{i}, y_{j}\right) \mid 1 \leq i \leq\right.$ $N-1,1 \leq j \leq N-1\}$, we consider the local cell

$$
\Omega_{i j}=\left\{(x, y) \mid\left(x-x_{i}\right)^{2}+\left(y-y_{j}\right)^{2} \leq H^{2}\right\},
$$

which is a disc with the center point $\mathbf{x}_{0}=\left(x_{i}, y_{j}\right)$ and radius $H$ in the domain $\Omega$. On the boundary of the local cell $\Omega_{i j}$, we take four points

$$
\mathbf{x}_{1}=\left(x_{i+1}, y_{j}\right), \quad \mathbf{x}_{2}=\left(x_{i}, y_{j+1}\right), \quad \mathbf{x}_{3}=\left(x_{i-1}, y_{j}\right), \quad \mathbf{x}_{4}=\left(x_{i}, y_{j-1}\right) .
$$


We try to find a numerical scheme for the numerical solution $u_{i j}$ at the points $\mathbf{x}_{0}, \mathbf{x}_{1}$, $\mathbf{x}_{2}, \mathbf{x}_{3}$, and $\mathbf{x}_{4}$. We point out that the essential difference between the one dimensional and two dimensional problems on the local cell is the boundary condition. For the one dimensional problem, the boundary only consists of two points. It is natural to assign the boundary conditions according to the equations (2.18)-(2.20) or the equations (2.29)-(2.34). For the two dimensional problem, the boundary is a circle. One needs to specify the boundary condition to make the local cell problem well-posed. In [8,9], Hou et al. proposed an oversampling technique to impose a boundary condition for local problems. In this paper, we proposed a boundary approximation technique to impose boundary conditions and solve the local cell problems.

First we present the boundary approximation technique based on the Fourier approximation. On the circle $\partial \Omega_{i j}$, assume the solution of (3.1) can be expanded as

$$
\begin{aligned}
\left.u^{\epsilon}(\mathbf{x})\right|_{\partial \Omega_{i j}} & =u^{\epsilon}(H, \theta) \\
& =\frac{a_{0}^{\epsilon}(H)}{2}+\sum_{n=1}^{\infty}\left(a_{n}^{\epsilon}(H) \cos (n \theta)+b_{n}^{\epsilon}(H) \sin (n \theta)\right),
\end{aligned}
$$

where $(H, \theta)$ is the ordered pair of polar coordinates of $\mathbf{x}=(x, y) \in \partial \Omega_{i j}$ with the pole at $\mathbf{x}_{0}$. The expansion (3.3) is the Fourier expansion of the function $u^{\epsilon}(H, \theta)$. On each local cell $\Omega_{i j}$, we consider the following cell problems

$$
\begin{aligned}
& \left\{\begin{array}{l}
-\nabla \cdot\left(A^{\epsilon}(\mathbf{x}) \nabla U_{0}^{\epsilon}(\mathbf{x})\right)+b^{\epsilon}(\mathbf{x}) U_{0}^{\epsilon}(\mathbf{x})=0, \quad \mathbf{x} \in \Omega_{i j}, \\
U_{0}^{\epsilon}(\mathbf{x})=1, \quad \mathbf{x} \in \partial \Omega_{i j} .
\end{array}\right. \\
& \left\{\begin{array}{l}
-\nabla \cdot\left(A^{\epsilon}(\mathbf{x}) \nabla U_{n}^{\epsilon}(\mathbf{x})\right)+b^{\epsilon}(\mathbf{x}) U_{n}^{\epsilon}(\mathbf{x})=0, \quad \mathbf{x} \in \Omega_{i j}, \\
U_{n}^{\epsilon}(\mathbf{x})=\cos (n \theta), \quad \mathbf{x} \in \partial \Omega_{i j}, \quad n=1,2,3, \cdots
\end{array}\right. \\
& \left\{\begin{array}{l}
-\nabla \cdot\left(A^{\epsilon}(\mathbf{x}) \nabla V_{n}^{\epsilon}(\mathbf{x})\right)+b^{\epsilon}(\mathbf{x}) V_{n}^{\epsilon}(\mathbf{x})=0, \quad \mathbf{x} \in \Omega_{i j}, \\
V_{n}^{\epsilon}(\mathbf{x})=\sin (n \theta), \quad \mathbf{x} \in \partial \Omega_{i j}, \quad n=1,2,3, \cdots
\end{array}\right. \\
& \left\{\begin{array}{l}
-\nabla \cdot\left(A^{\epsilon}(\mathbf{x}) \nabla U_{f}^{\epsilon}(\mathbf{x})\right)+b^{\epsilon}(\mathbf{x}) U_{f}^{\epsilon}(\mathbf{x})=f^{\epsilon}(\mathbf{x}), \quad \mathbf{x} \in \Omega_{i j}, \\
U_{f}^{\epsilon}(\mathbf{x})=0, \quad \mathbf{x} \in \partial \Omega_{i j} .
\end{array}\right.
\end{aligned}
$$

The local cell problems (3.4)-(3.7) have unique solutions separately. The solutions $U_{0}^{\epsilon}(\mathbf{x}), U_{n}^{\epsilon}(\mathbf{x}), V_{n}^{\epsilon}(\mathbf{x}), n=1,2,3, \cdots$ of the local cell problems (3.4)-(3.6) form a complete basis for the homogeneous equation of the problem (3.1)-(3.2) on the local cell $\Omega_{i j}$. The solution $U_{f}^{\epsilon}(\mathbf{x})$ comes form the inhomogeneous part, namely the force term $f^{\epsilon}(\mathbf{x})$.

Furthermore we can see that on the local cell $\Omega_{i j}$, the solution $u^{\epsilon}(\mathbf{x})$ of the problem (3.1)-(3.2) can be represented by

$$
\left.u^{\epsilon}(\mathbf{x})\right|_{\partial \Omega_{i j}}=\frac{1}{2} a_{0}^{\epsilon}(H) U_{0}^{\epsilon}(\mathbf{x})+\sum_{n=1}^{\infty}\left(a_{n}^{\epsilon}(H) U_{n}^{\epsilon}(\mathbf{x})+b_{n}^{\epsilon}(H) V_{n}^{\epsilon}(\mathbf{x})\right)+U_{f}^{\epsilon}(\mathbf{x}) .
$$

In practical computation, for instance in the five points numerical scheme, we only have the solution values at the boundary grid points $\mathbf{x}=\mathbf{x}_{1}, \mathbf{x}_{2}, \mathbf{x}_{3}, \mathbf{x}_{4}$. Therefore we take the solution in the space spanned by $U_{0}^{\epsilon}(\mathbf{x}), U_{1}^{\epsilon}(\mathbf{x}), V_{1}^{\epsilon}(\mathbf{x}), U_{2}^{\epsilon}(\mathbf{x})$, and $U_{f}^{\epsilon}(\mathbf{x})$ to approximate the solution $u^{\epsilon}(\mathbf{x})$. Recall that

$$
\left.U_{0}^{\epsilon}(\mathbf{x})\right|_{\partial \Omega_{i j}}=1,\left.\quad U_{1}^{\epsilon}(\mathbf{x})\right|_{\partial \Omega_{i j}}=\cos (\theta),\left.\quad V_{1}^{\epsilon}(\mathbf{x})\right|_{\partial \Omega_{i j}}=\sin (\theta),\left.\quad U_{2}^{\epsilon}(\mathbf{x})\right|_{\partial \Omega_{i j}}=\cos (2 \theta) .
$$


We define the basis functions as follows. Let

$$
\begin{aligned}
& P_{1}^{\epsilon}(\mathbf{x})=\frac{1}{4} U_{0}^{\epsilon}(\mathbf{x})+\frac{1}{2} U_{1}^{\epsilon}(\mathbf{x})+\frac{1}{4} U_{2}^{\epsilon}(\mathbf{x}), \\
& P_{2}^{\epsilon}(\mathbf{x})=\frac{1}{4} U_{0}^{\epsilon}(\mathbf{x})+\frac{1}{2} V_{1}^{\epsilon}(\mathbf{x})-\frac{1}{4} U_{2}^{\epsilon}(\mathbf{x}), \\
& P_{3}^{\epsilon}(\mathbf{x})=\frac{1}{4} U_{0}^{\epsilon}(\mathbf{x})-\frac{1}{2} U_{1}^{\epsilon}(\mathbf{x})+\frac{1}{4} U_{2}^{\epsilon}(\mathbf{x}), \\
& P_{4}^{\epsilon}(\mathbf{x})=\frac{1}{4} U_{0}^{\epsilon}(\mathbf{x})-\frac{1}{2} V_{1}^{\epsilon}(\mathbf{x})-\frac{1}{4} U_{2}^{\epsilon}(\mathbf{x}),
\end{aligned}
$$

then at the boundary grid points $\mathbf{x}=\mathbf{x}_{1}, \mathbf{x}_{2}, \mathbf{x}_{3}, \mathbf{x}_{4}$, we have $\left.P_{i}^{\epsilon}(\mathbf{x})\right|_{\mathbf{x}=\mathbf{x}_{j}}=\delta_{i j}$. On the local cell $\Omega_{i j}$, let

$$
u^{\epsilon, F}(\mathbf{x})=u^{\epsilon}\left(\mathbf{x}_{1}\right) P_{1}^{\epsilon}(\mathbf{x})+u^{\epsilon}\left(\mathbf{x}_{2}\right) P_{2}^{\epsilon}(\mathbf{x})+u^{\epsilon}\left(\mathbf{x}_{3}\right) P_{3}^{\epsilon}(\mathbf{x})+u^{\epsilon}\left(\mathbf{x}_{4}\right) P_{4}^{\epsilon}(\mathbf{x})+U_{f}^{\epsilon}(\mathbf{x})
$$

denote the approximate solution based on the Fourier approximation. Moreover, let $E^{\epsilon}(\mathbf{x})=u^{\epsilon}(\mathbf{x})-u^{\epsilon, F}(\mathbf{x})$ denote the error function. It is easy to see that $E^{\epsilon}(\mathbf{x})$ satisfies

$$
-\nabla \cdot\left(A^{\epsilon}(\mathbf{x}) \nabla E^{\epsilon}(\mathbf{x})\right)+b^{\epsilon}(\mathbf{x}) E^{\epsilon}(\mathbf{x})=0, \quad \mathbf{x} \in \Omega_{i j}
$$

and

$$
E^{\epsilon}\left(\mathbf{x}_{k}\right)=0, \quad \mathbf{x}_{k} \in \partial \Omega_{i j}, \quad k=1,2,3,4 .
$$

Therefore we use $u^{\epsilon, F}(\mathbf{x})$ to approximate the solution of the problem (3.1)-(3.2). Plugging $\mathbf{x}=\mathbf{x}_{0}$ into the Equation (3.10), we arrive at

$$
\begin{aligned}
u^{\epsilon}\left(\mathbf{x}_{0}\right) & \approx u^{\epsilon, F}\left(\mathbf{x}_{0}\right) \\
& =u^{\epsilon}\left(\mathbf{x}_{1}\right) P_{1}^{\epsilon}\left(\mathbf{x}_{0}\right)+u^{\epsilon}\left(\mathbf{x}_{2}\right) P_{2}^{\epsilon}\left(\mathbf{x}_{0}\right)+u^{\epsilon}\left(\mathbf{x}_{3}\right) P_{3}^{\epsilon}\left(\mathbf{x}_{0}\right)+u^{\epsilon}\left(\mathbf{x}_{4}\right) P_{4}^{\epsilon}\left(\mathbf{x}_{0}\right)+U_{f}^{\epsilon}\left(\mathbf{x}_{0}\right) .
\end{aligned}
$$

If we can get all the solutions of the local cell problems (3.4)-(3.7), then from (3.13) we immediately obtain the following discrete scheme for the problem (3.1)-(3.2) on the coarse grid $\Omega_{H}$ :

$$
\left\{\begin{array}{l}
u_{i j}=u_{i+1, j} p_{i j}^{1}+u_{i, j+1} p_{i j}^{2}+u_{i-1, j} p_{i j}^{3}+u_{i, j-1} p_{i j}^{4}+U_{i j}^{f}, \quad i, j=1, \cdots, N-1, \\
u_{i j}=0, \quad j=0 \quad \text { or } \quad j=N \\
u_{i j}=0, \quad i=0 \quad \text { or } \quad i=N
\end{array}\right.
$$

with $p_{i j}^{k}=P_{k}^{\epsilon}\left(\mathbf{x}_{0}\right)=P_{k}^{\epsilon}\left(x_{i}, y_{j}\right), k=1,2,3,4$, and $U_{i j}^{f}=U_{f}^{\epsilon}\left(\mathbf{x}_{0}\right)=U_{f}^{\epsilon}\left(x_{i}, y_{j}\right)$.

REMARK 3.1.1. The discrete maximum principle is very important for the stability of the numerical scheme. Numerical experiments indicate that discrete maximum principle still holds for the numerical scheme (3.14) on the condition that in (3.1) the operator $A^{\epsilon}(\mathbf{x})$ is positive definite with upper and lower bounds and the coefficient $b^{\epsilon}(\mathbf{x}) \geq 0$. However, the theoretical analysis about the stability of the numerical scheme (3.14) (based on the Fourier approximation on the boundary) is still open and will be our further consideration.

In general, we cannot obtain the exact solutions of the local problems (3.4)-(3.7). Therefore the coefficients $p_{i j}^{k}, k=1,2,3,4$, and $U_{i j}^{f}$ in the scheme (3.14) are unknown 
and we cannot directly use the discrete scheme (3.14) to obtain the numerical solution of the problem (3.1)-(3.2) on the coarse grid $\Omega_{H}$. We first need to solve the local problems (3.4)-(3.7) numerically to obtain the coefficients $p_{i j}^{k}, k=1,2,3,4$, and $U_{i j}^{f}$. Here we demonstrate this idea by solving the local problem (3.4) with the finite difference method.

Let $h=H / M$ denote the fine mesh size, where $H=1 / N$ is the coarse mesh size and $M, N$ are two positive integers. On each fixed local cell

$$
\Omega_{i j}=\left\{(x, y) \mid\left(x-x_{i}\right)^{2}+\left(y-y_{j}\right)^{2} \leq H^{2}\right\}, \quad i, j=1, \cdots N-1,
$$

we define a fine mesh grid by

$$
\Omega_{i j}^{h}=\left\{\left(x_{m}, y_{n}\right) \mid x_{m}=x_{i}+m h, y_{n}=y_{j}+n h, m^{2}+n^{2} \leq M^{2}, m, n=\cdots,-1,0,1, \cdots\right\},
$$

where $i, j$ are fixed.

Suppose $U=\left\{U_{m n}^{h} \mid m^{2}+n^{2} \leq M^{2}, m, n=\cdots,-1,0,1, \cdots\right\}$ is a grid function on the fine grid $\Omega_{i j}^{h}$, where $U_{m n}^{h}$ represents the approximate solution of $U_{0}^{\epsilon}(\mathbf{x})$ on the grid point $\left(x_{m}, y_{n}\right)$. The equation of the cell problem (3.4) can be rewritten as

$$
-\frac{\partial}{\partial x}\left(a_{1}^{\epsilon}(x, y) \frac{\partial U_{0}^{\epsilon}(x, y)}{\partial x}\right)-\frac{\partial}{\partial y}\left(a_{2}^{\epsilon}(x, y) \frac{\partial U_{0}^{\epsilon}(x, y)}{\partial y}\right)+b^{\epsilon}(x, y) U_{0}^{\epsilon}(x, y)=0 .
$$

At the interior mesh point $\left(x_{m}, y_{n}\right)$ in Figure 3.1, the Equation (3.15) is dis-

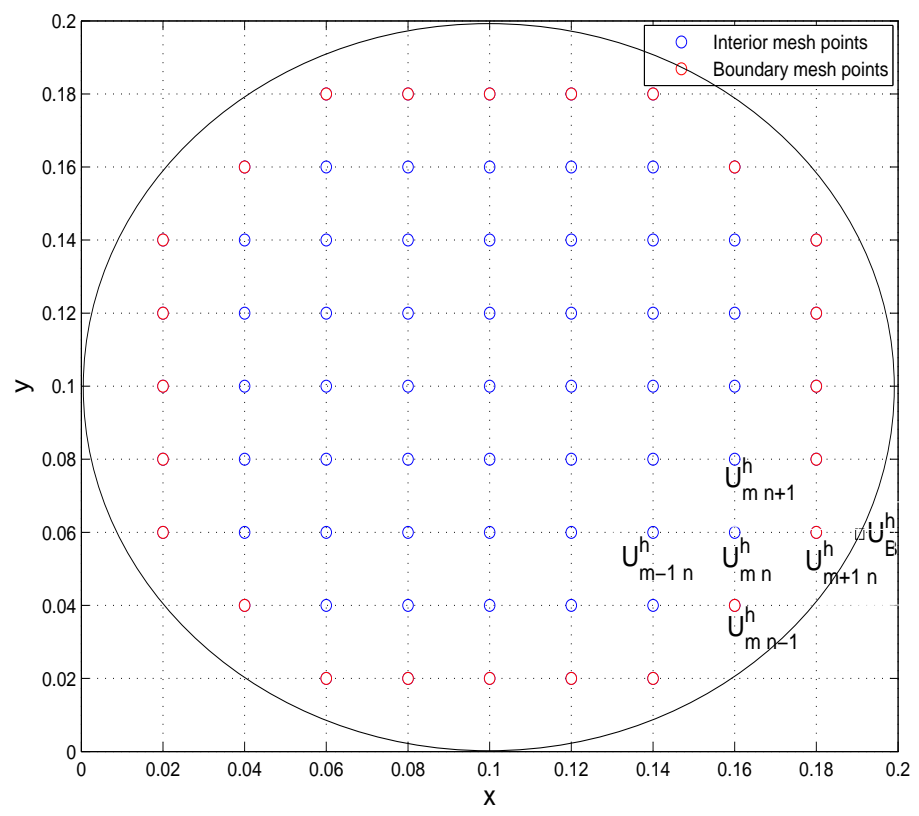

Figure 3.1. The fine grid of a local cell. 
cretized by the five points numerical scheme

$$
\begin{aligned}
& -\frac{a_{1}^{\epsilon}\left(x_{m+\frac{1}{2}}, y_{n}\right) U_{m+1, n}^{h}-\left(a_{1}^{\epsilon}\left(x_{m+\frac{1}{2}}, y_{n}\right)+a_{1}^{\epsilon}\left(x_{m-\frac{1}{2}}, y_{n}\right)\right) U_{m n}^{h}+a_{1}^{\epsilon}\left(x_{m-\frac{1}{2}}, y_{n}\right) U_{m-1, n}^{h}}{h^{2}} \\
& -\frac{a_{2}^{\epsilon}\left(x_{m}, y_{n+\frac{1}{2}}\right) U_{m, n+1}^{h}-\left(a_{2}^{\epsilon}\left(x_{m}, y_{n+\frac{1}{2}}\right)+a_{2}^{\epsilon}\left(x_{m}, y_{n-\frac{1}{2}}\right)\right) U_{m n}^{h}+a_{2}^{\epsilon}\left(x_{m}, y_{n-\frac{1}{2}}\right) U_{m, n-1}^{h}}{h^{2}} \\
& +b^{\epsilon}\left(x_{m}, y_{n}\right) U_{m n}^{h}=0 .
\end{aligned}
$$

At the boundary mesh points, we adopt the linear interpolation to obtain a numerical scheme; see [23]. For instance, at the point $\left(x_{m+1}, y_{n}\right)$ in Figure 3.1, we select a neighbor point $\left(x_{m}, y_{n}\right)$ such that the horizontal line through $\left(x_{m+1}, y_{n}\right)$ and $\left(x_{m}, y_{n}\right)$ cuts the boundary of $\Omega_{i j}$ at a point $\left(x_{B}, y_{n}\right)$, which is not a mesh point. For this boundary mesh point $\left(x_{m+1}, y_{n}\right)$, we define the numerical scheme

$$
U_{m+1, n}^{h}-\alpha_{m+1} U_{m, n}^{h}-\left(1-\alpha_{m+1}\right) U_{B}^{h}=0,
$$

where $\alpha_{m+1}=\frac{\left|x_{m+1}-x_{B}\right|}{h+\left|x_{m+1}-x_{B}\right|} \in\left(0, \frac{1}{2}\right]$ and $U_{B}^{h}=\left.U_{0}^{\epsilon}(\mathbf{x})\right|_{\mathbf{x}=\left(x_{B}, y_{n}\right)}$.

The numerical scheme obtained by combining (3.16) and (3.17) satisfies the discrete maximum principle [22]. Therefore we obtain the following result.

Lemma 3.1.2. The discrete scheme (3.16) and (3.17) for the local cell problem (3.4) has a unique solution $U_{m n}^{h}$.

REMARK 3.1.3. By solving the local cell problem (3.4), we obtain the grid function $U=\left\{U_{m n}^{h} \mid m^{2}+n^{2} \leq M^{2}, m, n=\cdots,-1,0,1, \cdots\right\}$ on $\Omega_{i j}^{h}$. We only need to save the value of the grid function at the center of $\Omega_{i j}^{h}$, namely, $U_{00}^{h}$ and pass it to the coarse grid solver (3.14). From discrete scheme (3.14) we can get the numerical solution of the problem (3.1)-(3.2) on the coarse grid $\Omega_{H}$. If we want more detailed structure of the solution, then we need to save the grid function $U$ on the fine grid $\Omega_{i j}^{h}$ and recover the numerical solution on the fine scale according to (3.10).

The local cell problems (3.5)-(3.7) can be discretized and solved by the same approach. We omit the details here.

3.2. Numerical scheme II. We proposed another multiscale tailored finite point scheme based on the Lagrange interpolation approximation. On the circle $\partial \Omega_{i j}$, we assume the solution of the Equation (3.1) can be approximated by

$$
\begin{aligned}
\left.u^{\epsilon}(\mathbf{x})\right|_{\partial \Omega_{i j}} & =u^{\epsilon}(H, \theta) \\
& \approx u^{\epsilon}(H, 0) L_{1}(\theta)+u^{\epsilon}\left(H, \frac{\pi}{2}\right) L_{2}(\theta)+u^{\epsilon}(H, \pi) L_{3}(\theta)+u^{\epsilon}\left(H, \frac{3 \pi}{2}\right) L_{4}(\theta),
\end{aligned}
$$

where $(H, \theta)$ is the ordered pair of polar coordinates of $\mathbf{x}=(x, y) \in \partial \Omega_{i j}$ with the pole at $\mathbf{x}_{0}$. The Lagrange interpolation base functions are defined by

$$
L_{1}(\theta)= \begin{cases}1-\frac{2 \theta}{\pi}, & 0 \leq \theta \leq \frac{\pi}{2}, \\ 0, & \frac{\pi}{2}<\theta<\frac{3 \pi}{2}, \\ \frac{2 \theta}{\pi}-3, & \frac{3 \pi}{2} \leq \theta<2 \pi\end{cases}
$$




$$
\begin{gathered}
L_{2}(\theta)= \begin{cases}\frac{2 \theta}{\pi}, & 0 \leq \theta \leq \frac{\pi}{2}, \\
2-\frac{2 \theta}{\pi}, & \frac{\pi}{2}<\theta \leq \pi, \\
0, & \pi<\theta<2 \pi,\end{cases} \\
L_{3}(\theta)= \begin{cases}0, & 0 \leq \theta<\frac{\pi}{2}, \\
\frac{2 \theta}{\pi}-1, & \frac{\pi}{2} \leq \theta \leq \pi, \\
3-\frac{2 \theta}{\pi}, & \pi<\theta \leq \frac{3 \pi}{2}, \\
0, & \frac{3 \pi}{2}<\theta<2 \pi,\end{cases} \\
L_{4}(\theta)= \begin{cases}0, & 0 \leq \theta<\pi, \\
\frac{2 \theta}{\pi}-2, & \pi \leq \theta \leq \frac{3 \pi}{2}, \\
4-\frac{2 \theta}{\pi}, & \frac{3 \pi}{2}<\theta<2 \pi\end{cases}
\end{gathered}
$$

On each local cell $\Omega_{i j}$, we consider the following cell problems:

$$
\begin{gathered}
\left\{\begin{array}{l}
-\nabla \cdot\left(A^{\epsilon}(\mathbf{x}) \nabla W_{k}^{\epsilon}(\mathbf{x})\right)+b^{\epsilon}(\mathbf{x}) W_{k}^{\epsilon}(\mathbf{x})=0, \quad \mathbf{x} \in \Omega_{i j}, \\
W_{k}^{\epsilon}(\mathbf{x})=L_{k}(\theta), \quad \mathbf{x} \in \partial \Omega_{i j}, \quad k=1,2,3,4 .
\end{array}\right. \\
\left\{\begin{array}{l}
-\nabla \cdot\left(A^{\epsilon}(\mathbf{x}) \nabla W_{f}^{\epsilon}(\mathbf{x})\right)+b^{\epsilon}(\mathbf{x}) W_{f}^{\epsilon}(\mathbf{x})=f^{\epsilon}(\mathbf{x}), \quad \mathbf{x} \in \Omega_{i j}, \\
W_{f}^{\epsilon}(\mathbf{x})=0, \quad \mathbf{x} \in \partial \Omega_{i j} .
\end{array}\right.
\end{gathered}
$$

The local cell problems (3.23) and (3.24) have unique solutions separately. Let

$$
u^{\epsilon, L}(\mathbf{x})=u^{\epsilon}\left(\mathbf{x}_{1}\right) W_{1}^{\epsilon}(\mathbf{x})+u^{\epsilon}\left(\mathbf{x}_{2}\right) W_{2}^{\epsilon}(\mathbf{x})+u^{\epsilon}\left(\mathbf{x}_{3}\right) W_{3}^{\epsilon}(\mathbf{x})+u^{\epsilon}\left(\mathbf{x}_{4}\right) W_{4}^{\epsilon}(\mathbf{x})+W_{f}^{\epsilon}(\mathbf{x})
$$

denote the approximate solution based on the Lagrange approximation. Moreover, let $E^{\epsilon}(\mathbf{x})=u^{\epsilon}(\mathbf{x})-u^{\epsilon, L}(\mathbf{x})$ denote the error function. It is easy to see that $E^{\epsilon}(\mathbf{x})$ also satisfies the equations (3.11)-(3.12). We use $u^{\epsilon, L}(\mathbf{x})$ to approximate the solution of the problem (3.1)-(3.2) on $\Omega_{i j}$. Plugging $\mathbf{x}=\mathbf{x}_{0}$ into the Equation (3.25), we arrive at

$$
\begin{aligned}
u^{\epsilon}\left(\mathbf{x}_{0}\right) & \approx u^{\epsilon, L}\left(\mathbf{x}_{0}\right) \\
& =u^{\epsilon}\left(\mathbf{x}_{1}\right) W_{1}^{\epsilon}\left(\mathbf{x}_{0}\right)+u^{\epsilon}\left(\mathbf{x}_{2}\right) W_{2}^{\epsilon}\left(\mathbf{x}_{0}\right)+u^{\epsilon}\left(\mathbf{x}_{3}\right) W_{3}^{\epsilon}\left(\mathbf{x}_{0}\right)+u^{\epsilon}\left(\mathbf{x}_{4}\right) W_{4}^{\epsilon}\left(\mathbf{x}_{0}\right)+W_{f}^{\epsilon}\left(\mathbf{x}_{0}\right) .
\end{aligned}
$$

If we can get all the solutions of the local cell problems (3.23) and (3.24), then from (3.26) we immediately obtain the following discrete scheme for the problem (3.1)-(3.2) on the coarse grid $\Omega_{H}$ :

$$
\left\{\begin{array}{l}
u_{i j}=u_{i+1, j} w_{i j}^{1}+u_{i, j+1} w_{i j}^{2}+u_{i-1, j} w_{i j}^{3}+u_{i, j-1} w_{i j}^{4}+w_{i j}^{f}, \quad i, j=1, \cdots, N-1 \\
u_{i j}=0, \quad j=0 \quad \text { or } \quad j=N \\
u_{i j}=0, \quad i=0 \quad \text { or } \quad i=N
\end{array}\right.
$$

with $w_{i j}^{k}=W_{k}^{\epsilon}\left(\mathbf{x}_{0}\right)=W_{k}^{\epsilon}\left(x_{i}, y_{j}\right), k=1,2,3,4$, and $w_{i j}^{f}=W_{f}^{\epsilon}\left(\mathbf{x}_{0}\right)=W_{f}^{\epsilon}\left(x_{i}, y_{j}\right)$. From the definition of the Lagrange interpolation basis functions (3.19)-(3.22) and the maximum principle [21], we obtain 
LEмma 3.2.1. In the discrete scheme (3.27) of the problem (3.1)-(3.2), we have the estimates

$$
\begin{aligned}
& 0<w_{i j}^{k}<1, \quad 1 \leq k \leq 4, \quad i, j=1, \cdots, N-1, \\
& 0<\sum_{k=1}^{4} w_{i j}^{k} \leq 1, \quad i, j=1, \cdots, N-1 .
\end{aligned}
$$

From Lemma 3.2.1, we immediately find that the matrix of the linear equation system (3.27) is diagonal dominant, thus it is invertible. Therefore the discrete scheme (3.27) for the problem (3.1)-(3.2) has a unique solution $U=\left\{u_{i j} \mid 0 \leq i \leq N, 0 \leq j \leq N\right\}$.

In general, we cannot obtain the analytic solution of the local problems (3.23) and (3.24). Therefore the coefficients $w_{i j}^{k}, k=1,2,3,4$, and $w_{i j}^{f}$ in the scheme (3.27) are unknown and we cannot directly use the discrete scheme (3.27) to obtain the numerical solution of the problem (3.1)-(3.2). The local problems (3.23) and (3.24) can be solved by the same numerical method proposed in the Section 3.1. We omit the details here.

In the multiscale tailored finite point method (MsTFPM), the construction of the base functions and load functions is fully decoupled from cell to cell; thus, this method is perfectly parallel and is naturally adapted to massively parallel computers. For the same reason, this method has the ability to handle multiscale problems with extremely large degrees of freedom, which are intractable by conventional finite element method (FEM) or finite difference method (FDM). For example, let $N$ be the partition number of the coarse grid in each spatial direction, and let $M$ be the partition number of the fine grid in each direction for solving the local cell problem. Let $d$ be the dimension of the problem $\left(\mathrm{d}=1,2\right.$ in this paper). Then there are total $(N M)^{d}$ unknowns at the fine grid level. For a traditional FEM or FDM, the computer memory needed for solving the problem on the fine grid is $O\left(M^{d} N^{d}\right)$. In contrast, the multiscale tailored finite point method (MsTFPM) requires only $O\left(M^{d}+N^{d}\right)$ amount of memory. In this sense, the multiscale tailored finite point method (MsTFPM) has the same advantage as the multiscale finite element methods (MsFEM) [8].

\section{Numerical example}

In this section, we present both the one- and two-dimensional examples to demonstrate the efficiency and accuracy of the multiscale tailored finite point method (MsTFPM). Especially, we want to show that our method can capture the small scales information on a very coarse mesh grid.

4.1. Examples of one dimensional problems. In this section, we consider the numerical solution to the one dimensional multiscale elliptic problem with highly oscillatory or rough coefficients. The problem is given by

$$
\left\{\begin{array}{l}
-\left(a^{\epsilon}(x) u^{\epsilon}(x)^{\prime}\right)^{\prime}+b^{\epsilon}(x) u^{\epsilon}(x)=f^{\epsilon}(x), \quad x \in(0,1), \\
u^{\epsilon}(0)=0, \quad u^{\epsilon}(1)=0 .
\end{array}\right.
$$

Example 4.1. Consider the one dimensional multiscale elliptic problem (4.1) with

$$
a^{\epsilon}(x)=\frac{1}{2+P \cos (x / \epsilon)}, \quad b^{\epsilon}(x)=0, \quad f^{\epsilon}(x)=1,
$$

where $\epsilon=0.01$ and $P=1.99$. This example is a model problem from the book [14]. The coefficient $a^{\epsilon}(x)$ is periodic in $2 \pi \epsilon$ and has clear scale separation; see the Figure 4.1.1. 


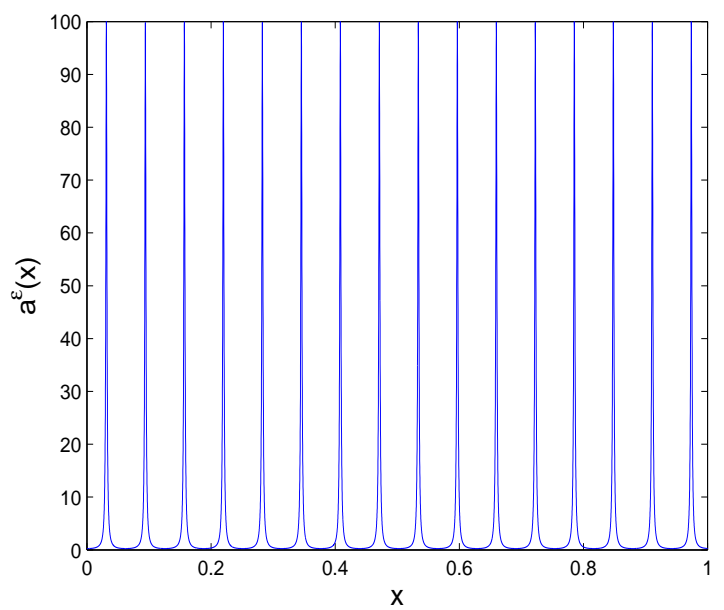

Figure 4.1.1. The coefficient $a^{\epsilon}(x)$ of Example 4.1.
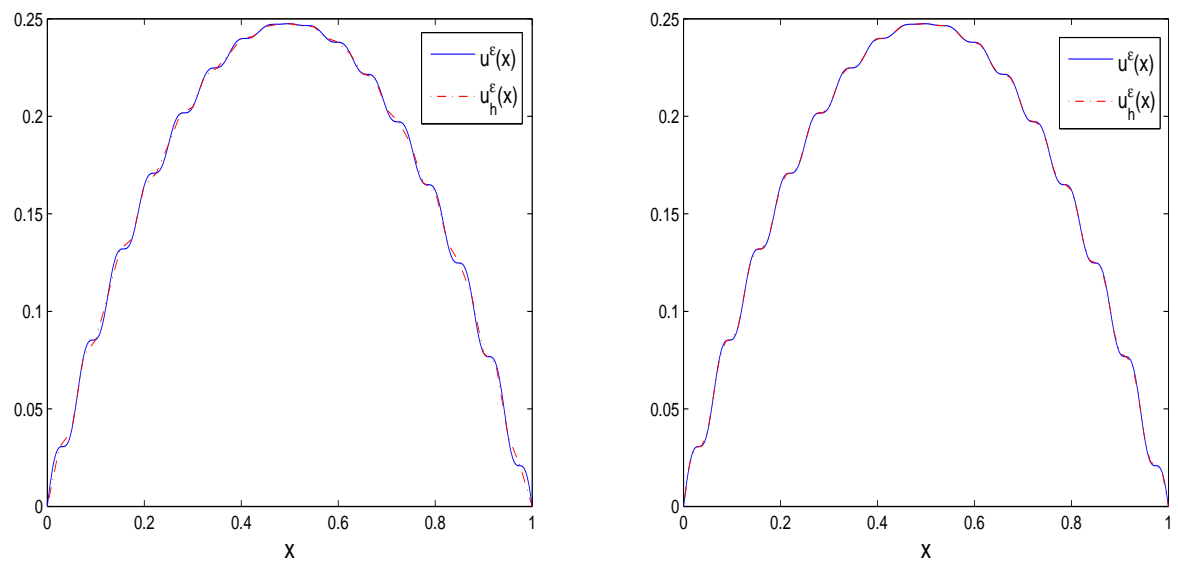

Figure 4.1.2. The numerical solution of Example 4.1. Left is for $N=20, M=10$, and right is for $N=40, M=10$.

\begin{tabular}{llllll}
\hline Coarse grid & Fine grid & $L^{\infty}$ error & \multicolumn{3}{l}{$L^{2}$ error } \\
\hline $\mathrm{N}=10$ & $\mathrm{M}=10$ & $2.285 \mathrm{e}-3$ & $\cdots$ & $3.196 \mathrm{e}-4$ & $\cdots$ \\
$\mathrm{N}=20$ & $\mathrm{M}=10$ & $5.179 \mathrm{e}-4$ & 2.100 & $7.259 \mathrm{e}-5$ & 2.098 \\
$\mathrm{~N}=40$ & $\mathrm{M}=10$ & $1.264 \mathrm{e}-4$ & 2.023 & $1.653 \mathrm{e}-5$ & 2.095 \\
$\mathrm{~N}=80$ & $\mathrm{M}=10$ & $3.547 \mathrm{e}-5$ & 1.888 & $4.152 \mathrm{e}-6$ & 1.995 \\
$\mathrm{~N}=160$ & $\mathrm{M}=10$ & $9.089 \mathrm{e}-6$ & 1.975 & $1.033 \mathrm{e}-6$ & 2.004 \\
$\mathrm{~N}=320$ & $\mathrm{M}=10$ & $2.284 \mathrm{e}-6$ & 1.994 & $2.553 \mathrm{e}-7$ & 2.012 \\
$\mathrm{~N}=640$ & $\mathrm{M}=10$ & $5.438 \mathrm{e}-7$ & 2.049 & $6.077 \mathrm{e}-8$ & 2.049 \\
\hline
\end{tabular}

TABLE 4.1. Numerical results of the method scheme I for Example 4.1. 


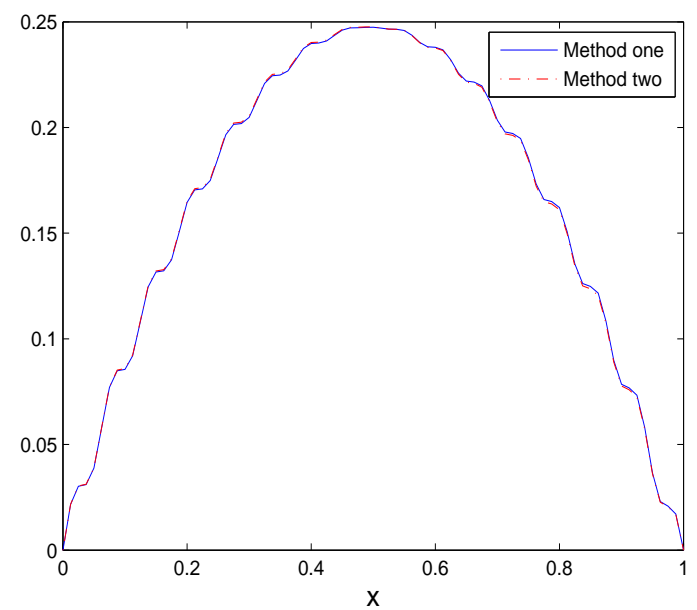

FiguRE 4.1.3. The comparison between the numerical schemes I and II. The solid line is for the numerical scheme $I$ and the dashed line is for the numerical scheme II. $N=40$, $M=10$.

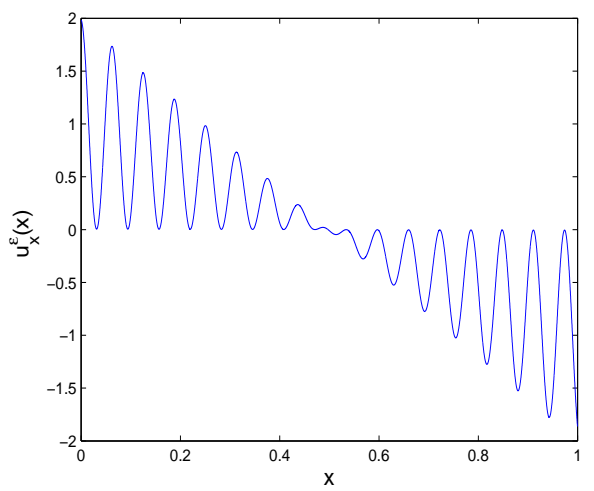

FiguRE 4.1.4. The derivative of the numerical solution $u^{\epsilon}(x)$ for Example 4.1. 


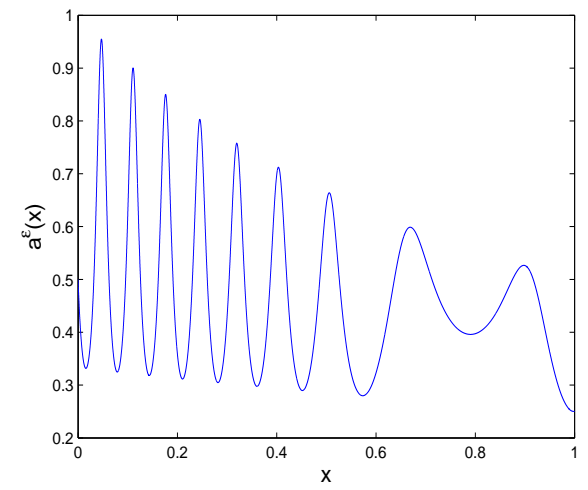

Figure 4.2.1. The coefficient $a^{\epsilon}(x)$ of Example 4.2.
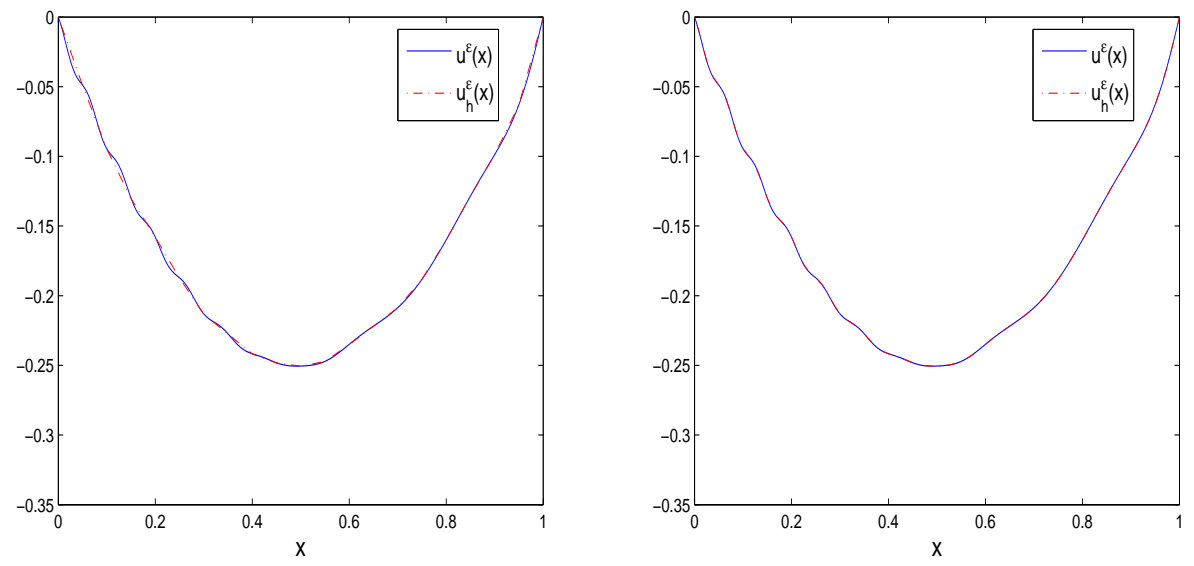

Figure 4.2.2. The numerical solutions of Example 4.2. Left is for $N=20, M=10$, and right is for $N=40, M=10$. 


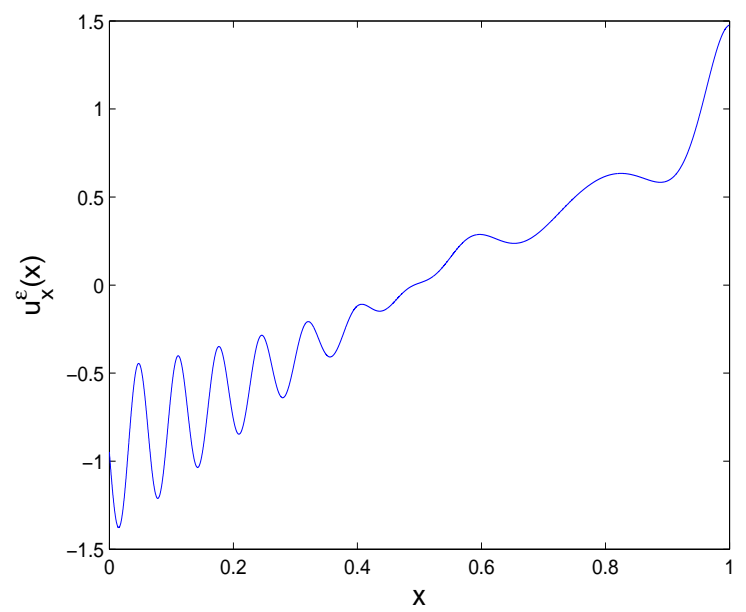

FIGURE 4.2.3. The derivative of the solution $u^{\epsilon}(x)$ for Example 4.2.

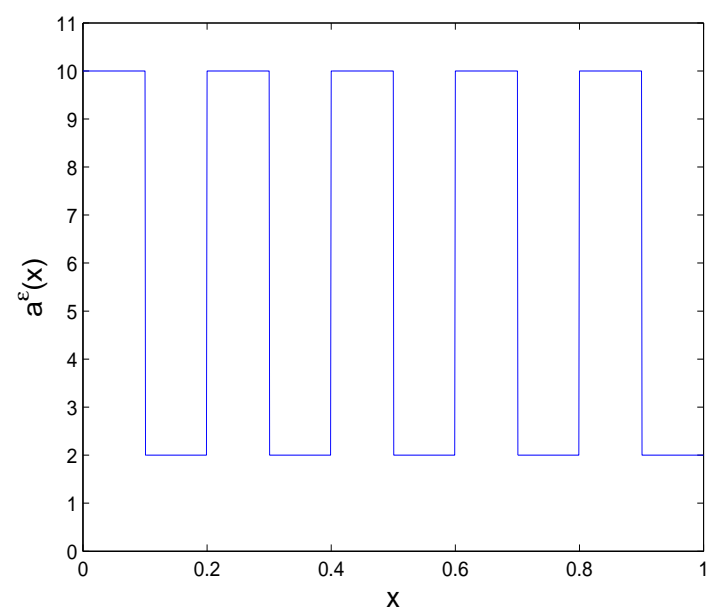

Figure 4.3.1. The coefficient $a^{\epsilon}(x)$ of Example 4.3. 


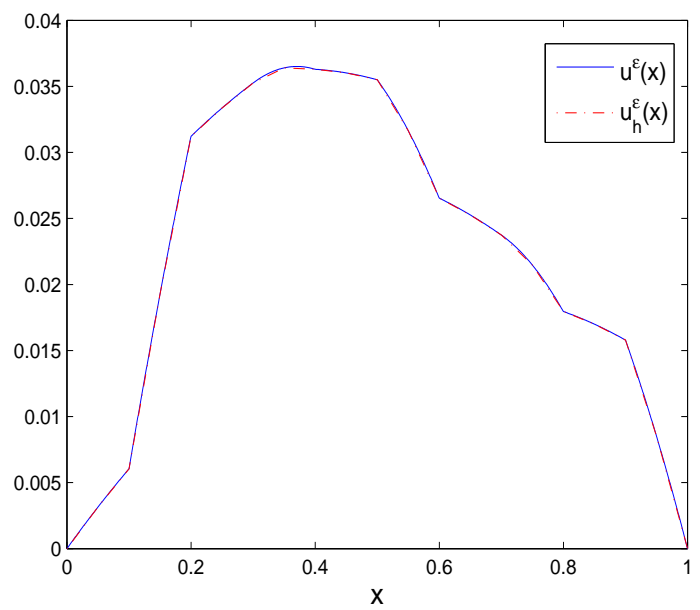

FigurE 4.3.2. The numerical solution of Example 4.3. $N=10, M=10$.

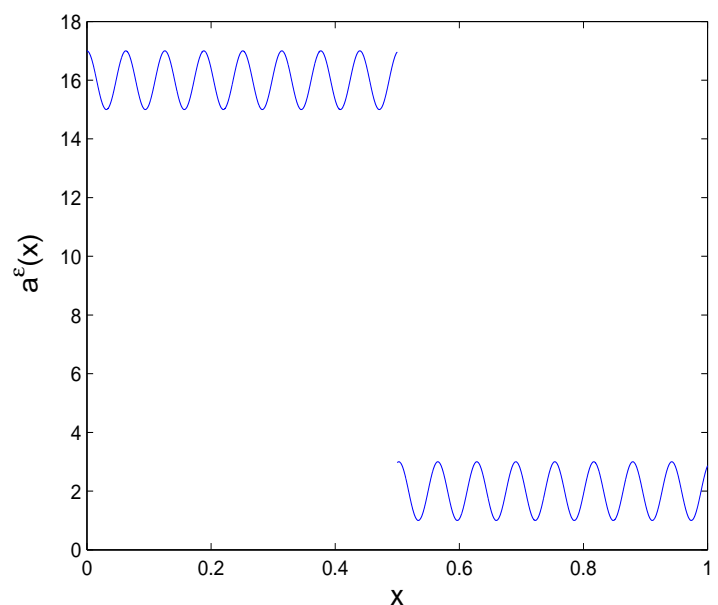

FiguRE 4.4.1. The coefficient $a^{\epsilon}(x)$ of Example 4.4. 


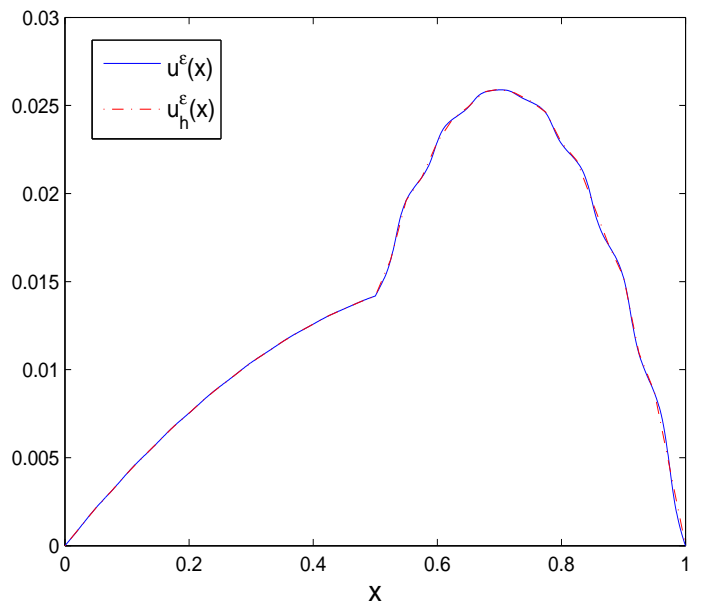

FiguRE 4.4.1. The numerical solution of Example 4.4. $N=20, M=10$.
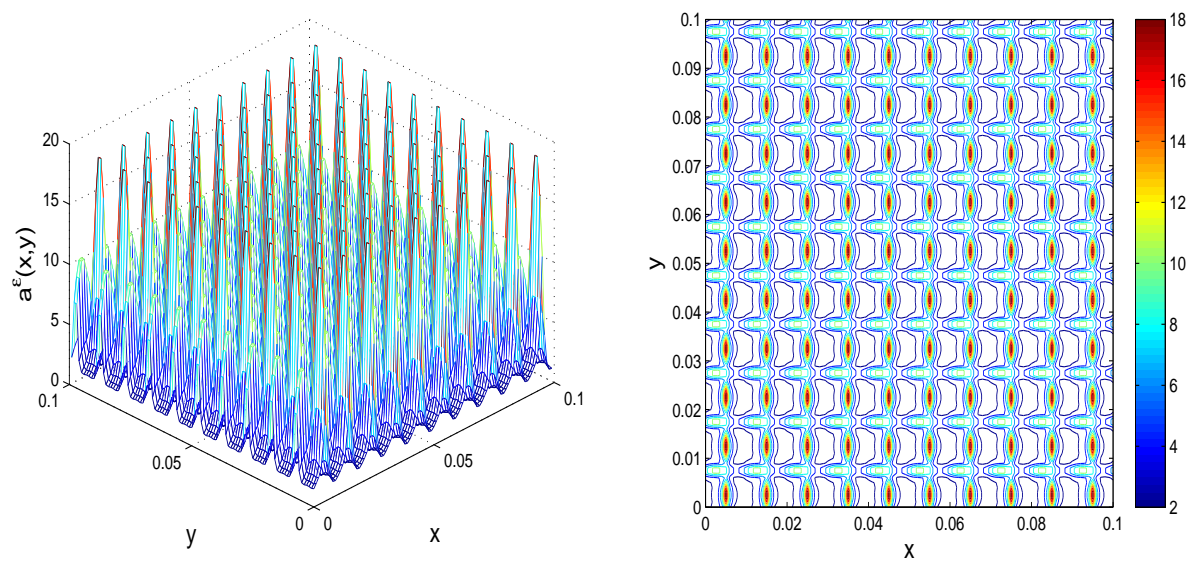

FiguRE 4.5.1. The coefficient $a^{\epsilon}(x, y)$ of Example 4.5 in the domain $[0,0.1] \times[0,0.1]$. 


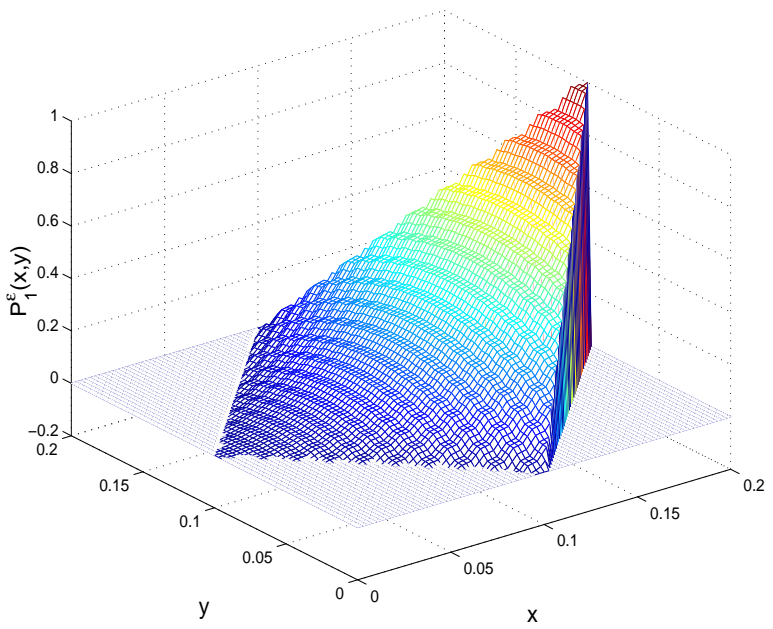

FIGURE 4.5.2. Surface plot of a typical local basis function.

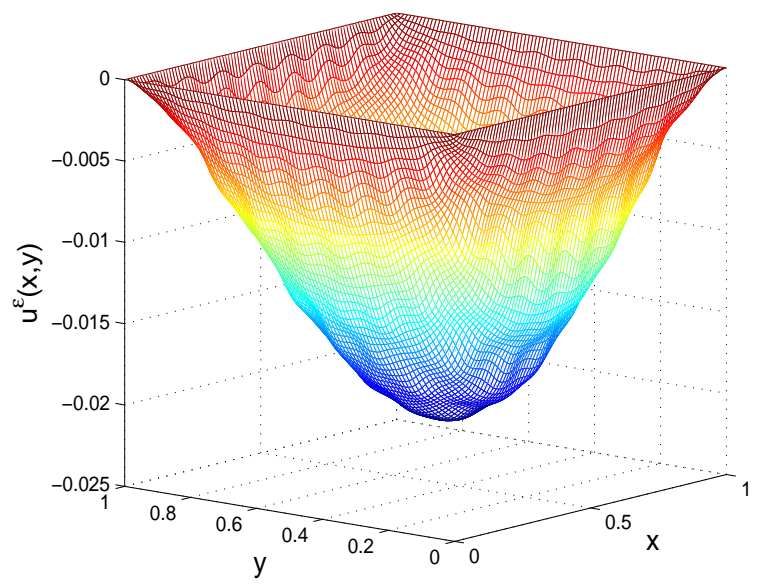

FIgURE 4.5.3. The numerical solution of Example 4.5 (surface plot). 


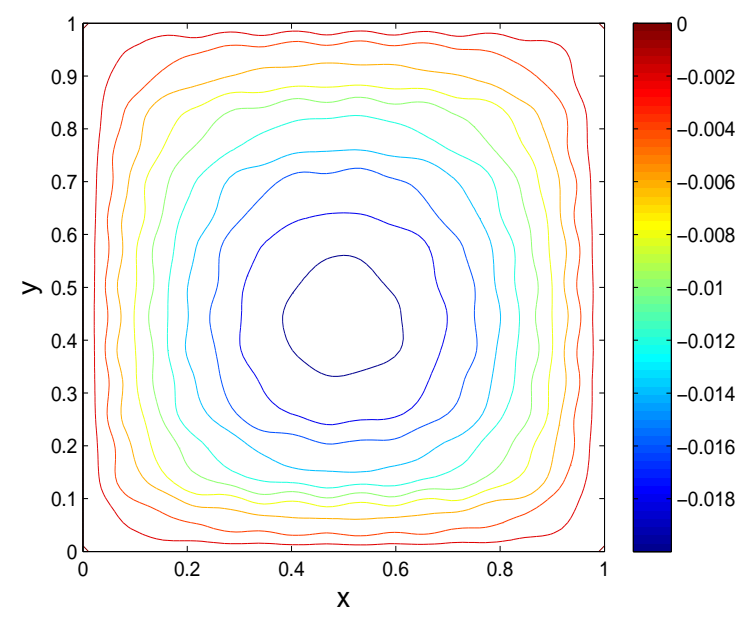

FiguRE 4.5.4. The numerical solution of Example 4.5 (contour plot).
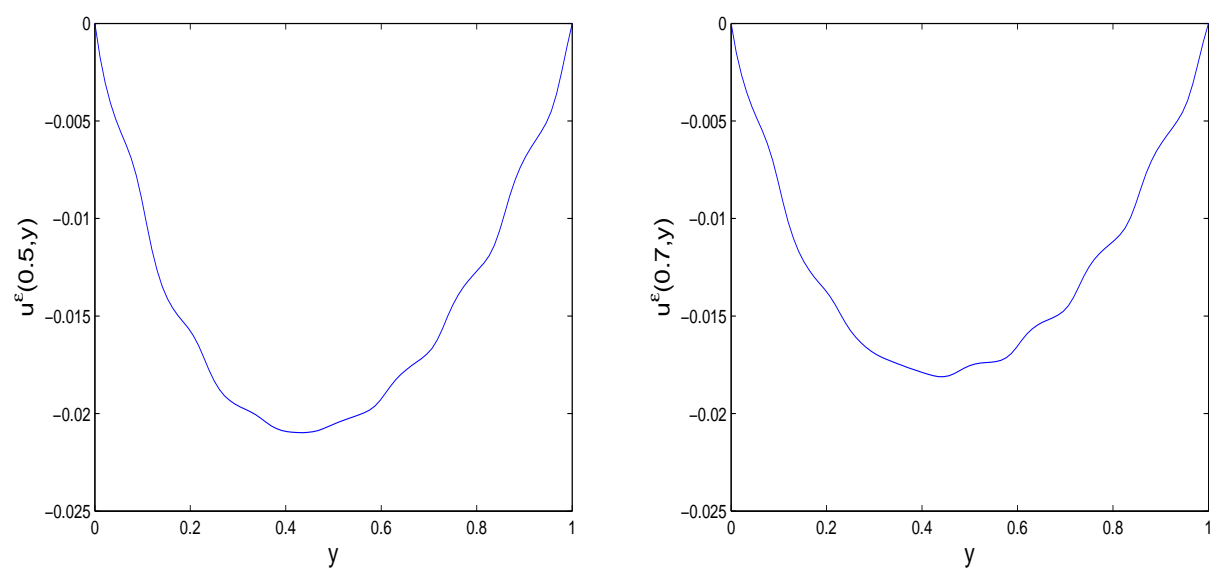

FiguRE 4.5.5. The numerical solutions for Example 4.5 (slice plot).

The numerical results of Example 4.1 are shown in Figure 4.1.2 -Figure 4.1.4 and Table 4.1. In Figure 4.1.2, we plot the numerical solutions obtained by the numerical scheme one on different mesh grids. Figure 4.1.2 shows that our method can approximate the 'exact' solution on very coarse mesh. Here the 'exact' solution is obtained on the fine mesh with $h=1 / 25600$. In Figure 4.1.3, we compare the performance of the numerical scheme I and numerical scheme II. In Figure 4.1.4, we plot the derivative of function $u_{h}^{\epsilon}(x)$. One can see that the solution of the Example 4.1 itself is oscillatory in the level of $O(\epsilon)$, but its derivative function oscillates in the level of $O(1)$. In Table 4.1, we show the numerical errors and convergence rate of the numerical scheme I, which indicates that our method has second order convergence 

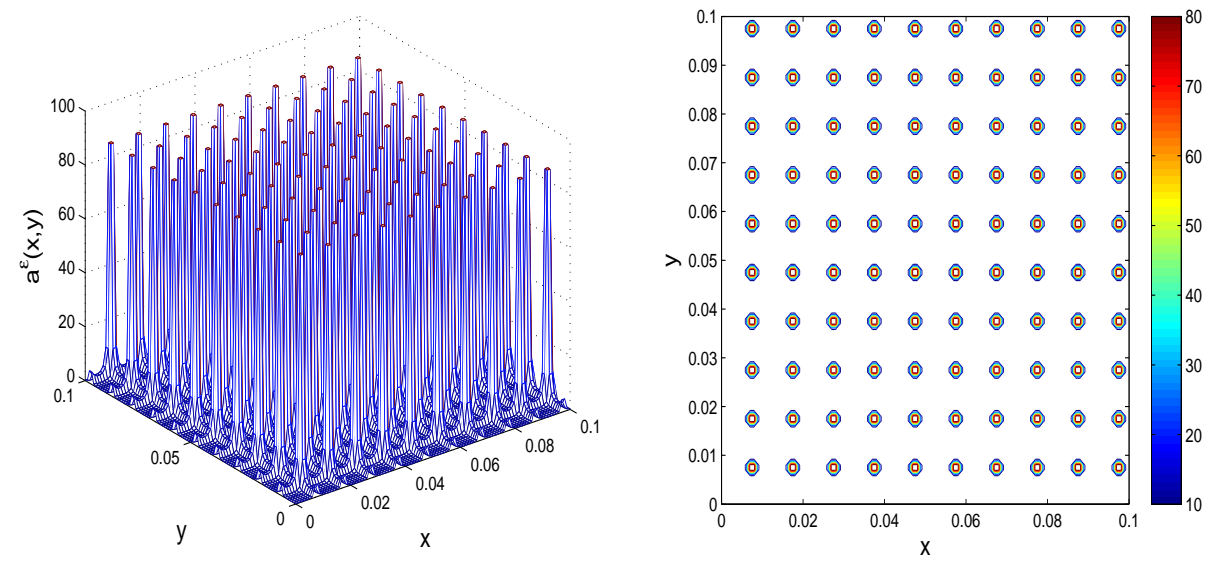

Figure 4.6.1. The coefficient $a^{\epsilon}(x, y)$ of Example 4.6 in the domain $[0,0.1] \times[0,0.1]$.

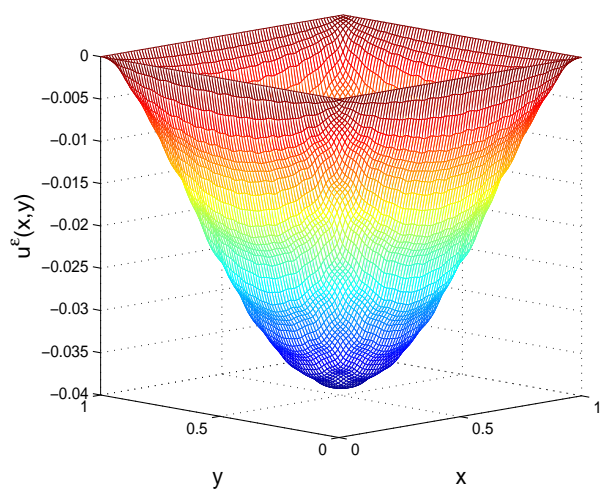

FIGURE 4.6.2. The numerical solution of Example 4.6 (surface plot).

in the $L^{\infty}$ and $L^{2}$ norms.

EXAMPLE 4.2. Consider the one dimensional multiscale elliptic problem (4.1) with

$$
a^{\epsilon}(x)=\frac{1}{2+x+\sin \left(\frac{\sin x}{\epsilon} \cos (x)\right)}, \quad b^{\epsilon}(x)=0, \quad f^{\epsilon}(x)=-\cos (x),
$$

where $\epsilon=0.01$. In this example, the coefficient $a^{\epsilon}(x)$ is not periodic and there is no clear scale separation; see Figure 4.2.1. The numerical results of Example 4.2 are shown in Figure 4.2.2, Figure 4.2.3, and Table 4.2. In Figure 4.2.2, we show the numerical solutions obtained by the numerical scheme I on different mesh grids. In Figure 4.2.3, we plot the derivative of function $u_{h}^{\epsilon}(x)$. In Table 4.2, we show the numerical errors and convergence rate of the numerical scheme I, which indicates that our method has second order convergence in $L^{\infty}$ and $L^{2}$ norms. Here the 'exact' 


\begin{tabular}{llllll}
\hline Coarse grid & Fine grid & $L^{\infty}$ error & \multicolumn{3}{l}{$L^{2}$ error } \\
\hline $\mathrm{N}=10$ & $\mathrm{M}=10$ & $1.393 \mathrm{e}-3$ & $\cdots$ & $1.927 \mathrm{e}-4$ & $\ldots$ \\
$\mathrm{N}=20$ & $\mathrm{M}=10$ & $3.188 \mathrm{e}-4$ & 2.090 & $4.180 \mathrm{e}-5$ & 2.147 \\
$\mathrm{~N}=40$ & $\mathrm{M}=10$ & $8.630 \mathrm{e}-5$ & 1.922 & $1.037 \mathrm{e}-5$ & 2.007 \\
$\mathrm{~N}=80$ & $\mathrm{M}=10$ & $2.144 \mathrm{e}-5$ & 2.006 & $2.575 \mathrm{e}-6$ & 2.006 \\
$\mathrm{~N}=160$ & $\mathrm{M}=10$ & $5.816 \mathrm{e}-6$ & 1.919 & $6.411 \mathrm{e}-7$ & 2.004 \\
$\mathrm{~N}=320$ & $\mathrm{M}=10$ & $1.436 \mathrm{e}-6$ & 2.012 & $1.583 \mathrm{e}-7$ & 2.012 \\
$\mathrm{~N}=640$ & $\mathrm{M}=10$ & $3.419 \mathrm{e}-7$ & 2.049 & $3.769 \mathrm{e}-8$ & 2.049 \\
\hline
\end{tabular}

TABLE 4.2. Numerical results of the method scheme I for Example 4.2.

\begin{tabular}{llllll}
\hline Coarse grid & Fine grid & $L^{\infty}$ error & & $L^{2}$ error & \\
\hline $\mathrm{N}=10$ & $\mathrm{M}=10$ & $6.639 \mathrm{e}-11$ & $\cdots$ & $1.381 \mathrm{e}-11$ & $\cdots$ \\
$\mathrm{N}=20$ & $\mathrm{M}=10$ & $6.639 \mathrm{e}-11$ & $\cdots$ & $1.366 \mathrm{e}-11$ & $\cdots$ \\
$\mathrm{N}=40$ & $\mathrm{M}=10$ & $6.660 \mathrm{e}-11$ & $\cdots$ & $1.362 \mathrm{e}-11$ & $\cdots$ \\
$\mathrm{N}=80$ & $\mathrm{M}=10$ & $6.655 \mathrm{e}-11$ & $\cdots$ & $1.360 \mathrm{e}-11$ & $\cdots$ \\
\hline
\end{tabular}

TABLE 4.3. Numerical results of the method scheme II for the Example 4.3.

solution is still obtained on the fine mesh with $h=1 / 25600$. From these results, we see that our method has good performance for the multiscale elliptic problem with nonperiodic coefficient:

ExAmple 4.3. Consider the one dimensional multiscale elliptic problem (4.1) with

$$
\begin{gathered}
a^{\epsilon}(x)=\left\{\begin{array}{l}
10, \quad x_{i-1}<x<\frac{x_{i-1}+x_{i}}{2}, \\
2, \quad \frac{x_{i-1}+x_{i}}{2}<x<x_{i}, \quad i=0, \cdots, 5,
\end{array}\right. \\
b^{\epsilon}(x)=0, \quad f^{\epsilon}(x)=1,
\end{gathered}
$$

where $x_{i}=i / 5, i=0, \cdots, 5$. This example is introduced in the paper [4]. In this example the coefficient $a^{\epsilon}(x)$ is discontinuous; see Figure 4.3.1. Let $D=\left\{\bar{x}_{i}=i / 10 \mid i=1, \cdots, 9\right\}$ denote the discontinuous point set. We use the numerical scheme II to solve this problem. It should be pointed out that we partition the domain $[0,1]$ in such a way that the interface set contains the discontinuous point set $D$ as a subset.

The numerical results of Example 4.3 are shown in Figure 4.3.2 and Table 4.3. From these results, we see that our method can achieve machine accuracy for Example 4.3 on the condition that all the discontinuous points are contained in the interface points of the numerical scheme II.

ExAmple 4.4. Consider the one dimensional multiscale elliptic problem (4.1) with

$$
\begin{gathered}
a^{\epsilon}(x)=\left\{\begin{array}{l}
16+\cos (x / \epsilon), \quad 0<x<0.5, \\
2+\cos (x / \epsilon), \quad 0.5<x<1,
\end{array}\right. \\
b^{\epsilon}(x)=0, \quad f^{\epsilon}(x)=1,
\end{gathered}
$$




\begin{tabular}{llllll}
\hline Coarse grid & Fine grid & $L^{\infty}$ error & \multicolumn{3}{l}{$L^{2}$ error } \\
\hline $\mathrm{N}=10$ & $\mathrm{M}=10$ & $2.844 \mathrm{e}-3$ & $\cdots$ & $4.871 \mathrm{e}-4$ & $\cdots$ \\
$\mathrm{N}=20$ & $\mathrm{M}=10$ & $2.110 \mathrm{e}-4$ & 3.671 & $3.005 \mathrm{e}-5$ & 4.026 \\
$\mathrm{~N}=40$ & $\mathrm{M}=10$ & $6.136 \mathrm{e}-5$ & 1.854 & $8.392 \mathrm{e}-6$ & 1.892 \\
$\mathrm{~N}=80$ & $\mathrm{M}=10$ & $1.206 \mathrm{e}-5$ & 2.255 & $2.261 \mathrm{e}-6$ & 1.926 \\
$\mathrm{~N}=160$ & $\mathrm{M}=10$ & $1.826 \mathrm{e}-6$ & 2.569 & $2.792 \mathrm{e}-7$ & 2.845 \\
$\mathrm{~N}=320$ & $\mathrm{M}=10$ & $4.549 \mathrm{e}-7$ & 2.003 & $6.938 \mathrm{e}-8$ & 2.006 \\
$\mathrm{~N}=640$ & $\mathrm{M}=10$ & $1.084 \mathrm{e}-7$ & 2.048 & $1.653 \mathrm{e}-8$ & 2.048 \\
\hline
\end{tabular}

TABLE 4.4. Numerical results of the method scheme II for the Example 4.4.

\begin{tabular}{llllll}
\hline Coarse grid & Fine grid & $L^{\infty}$ error & & $L^{2}$ error & \\
\hline $\mathrm{N}=5$ & $\mathrm{M}=40$ & $1.911 \mathrm{e}-2$ & $\cdots$ & $3.210 \mathrm{e}-4$ & $\cdots$ \\
$\mathrm{N}=10$ & $\mathrm{M}=40$ & $6.843 \mathrm{e}-3$ & 1.671 & $8.985 \mathrm{e}-5$ & 1.890 \\
$\mathrm{~N}=20$ & $\mathrm{M}=40$ & $2.945 \mathrm{e}-3$ & 1.524 & $3.974 \mathrm{e}-5$ & 1.503 \\
$\mathrm{~N}=40$ & $\mathrm{M}=40$ & $8.355 \mathrm{e}-4$ & 1.877 & $1.006 \mathrm{e}-5$ & 1.987 \\
\hline
\end{tabular}

TABLE 4.5. Numerical results of the Fourier approximation for the Example 4.5.

\begin{tabular}{llllll}
\hline Coarse grid & Fine grid & $L^{\infty}$ error & & $L^{2}$ error & \\
\hline $\mathrm{N}=5$ & $\mathrm{M}=40$ & $1.899 \mathrm{e}-2$ & $\cdots$ & $3.211 \mathrm{e}-4$ & $\cdots$ \\
$\mathrm{N}=10$ & $\mathrm{M}=40$ & $6.685 \mathrm{e}-3$ & 1.685 & $8.969 \mathrm{e}-5$ & 1.892 \\
$\mathrm{~N}=20$ & $\mathrm{M}=40$ & $2.858 \mathrm{e}-3$ & 1.529 & $3.899 \mathrm{e}-5$ & 1.516 \\
$\mathrm{~N}=40$ & $\mathrm{M}=40$ & $7.050 \mathrm{e}-4$ & 2.013 & $9.340 \mathrm{e}-6$ & 2.043 \\
\hline
\end{tabular}

TABlE 4.6. Numerical results of the Lagrange interpolation approximation for the Example 4.5.

where $\epsilon=0.01$. In this case, the coefficient $a^{\epsilon}(x)$ is discontinuous and oscillatory; see Figure 4.4.1. We use the numerical scheme II to solve this problem. We partition the domain $[0,1]$ in such a way that the interface point set contains the discontinuous point $x=0.5$.

The numerical results of Example 4.4 are shown in Figure 4.4.2 and Table 4.4. We can see that our method can approximate the 'exact' solution on very coarse mesh and have second order convergence in $L^{\infty}$ and $L^{2}$ norms. Here the 'exact' solution is obtained on the fine mesh with $h=1 / 25600$.

4.2. Examples of two dimensional problems. We now consider the two dimensional elliptic multiscale problem on the domain $\Omega=[0,1] \times[0,1]$ given by

$$
\left\{\begin{array}{l}
-\nabla \cdot\left(A^{\epsilon}(\mathbf{x}) \nabla u^{\epsilon}(\mathbf{x})\right)+b^{\epsilon}(\mathbf{x}) u^{\epsilon}(\mathbf{x})=f^{\epsilon}(\mathbf{x}), \quad \mathbf{x}=(x, y) \in \Omega \\
u^{\epsilon}(\mathbf{x})=0, \quad \mathbf{x}=(x, y) \in \partial \Omega
\end{array}\right.
$$

where the operator $A^{\epsilon}(\mathbf{x})=a^{\epsilon}(x, y) I_{2 \times 2}$ is a diagonal matrix function with $0<\alpha \leq$ $a^{\epsilon}(x, y)<\beta$, the coefficient $b^{\epsilon}(\mathbf{x})=b^{\epsilon}(x, y) \geq 0$, and the force term $f^{\epsilon}(\mathbf{x})=f^{\epsilon}(x, y)$.

ExAmple 4.5. Consider the two dimensional multiscale elliptic problem (4.2) with

$$
\begin{aligned}
& a^{\epsilon}(x, y)=\frac{2+P \sin \left(\frac{2 \pi x}{\epsilon}\right)}{2+P \sin \left(\frac{2 \pi y}{\epsilon}\right)}+\frac{2+\sin \left(\frac{2 \pi y}{\epsilon}\right)}{2+P \sin \left(\frac{2 \pi x}{\epsilon}\right)} \\
& b^{\epsilon}(x, y)=0, \quad f^{\epsilon}(x, y)=-1
\end{aligned}
$$




\begin{tabular}{llllll}
\hline Coarse grid & Fine grid & $L^{\infty}$ error & \multicolumn{3}{c}{$L^{2}$ error } \\
\hline $\mathrm{N}=5$ & $\mathrm{M}=40$ & $2.658 \mathrm{e}-1$ & $\ldots$ & $3.989 \mathrm{e}-3$ & $\cdots$ \\
$\mathrm{N}=10$ & $\mathrm{M}=40$ & $1.183 \mathrm{e}-1$ & 1.168 & $1.671 \mathrm{e}-3$ & 1.255 \\
$\mathrm{~N}=20$ & $\mathrm{M}=40$ & $2.290 \mathrm{e}-2$ & 2.369 & $3.187 \mathrm{e}-4$ & 2.391 \\
$\mathrm{~N}=40$ & $\mathrm{M}=40$ & $1.025 \mathrm{e}-2$ & 1.159 & $1.475 \mathrm{e}-4$ & 1.112 \\
\hline
\end{tabular}

TABLE 4.7. Numerical results of the Fourier approximation for the Example 4.6.

\begin{tabular}{llllll}
\hline Coarse grid & Fine grid & $L^{\infty}$ error & \multicolumn{3}{c}{$L^{2}$ error } \\
\hline $\mathrm{N}=5$ & $\mathrm{M}=40$ & $2.654 \mathrm{e}-1$ & $\cdots$ & $3.978 \mathrm{e}-3$ & $\cdots$ \\
$\mathrm{N}=10$ & $\mathrm{M}=40$ & $1.132 \mathrm{e}-1$ & 1.229 & $1.683 \mathrm{e}-3$ & 1.241 \\
$\mathrm{~N}=20$ & $\mathrm{M}=40$ & $2.196 \mathrm{e}-2$ & 2.366 & $3.163 \mathrm{e}-4$ & 2.411 \\
$\mathrm{~N}=40$ & $\mathrm{M}=40$ & $9.810 \mathrm{e}-2$ & 1.163 & $1.421 \mathrm{e}-4$ & 1.154 \\
\hline
\end{tabular}

TABLE 4.8. Numerical results of the Lagrange interpolation approximation for the Example 4.6.

where $\epsilon=0.01$ and $P=1.8$. This example is a model problem from the paper [8]. In this example, the coefficient $a^{\epsilon}(x, y)$ is periodic in $\epsilon$ in each direction and has clear scale separation. In Figure 4.5.1, we only give the surface plot and contour plot of the coefficient $a^{\epsilon}(x, y)$ on a small domain $[0,0.1] \times[0,0.1]$ for demonstration.

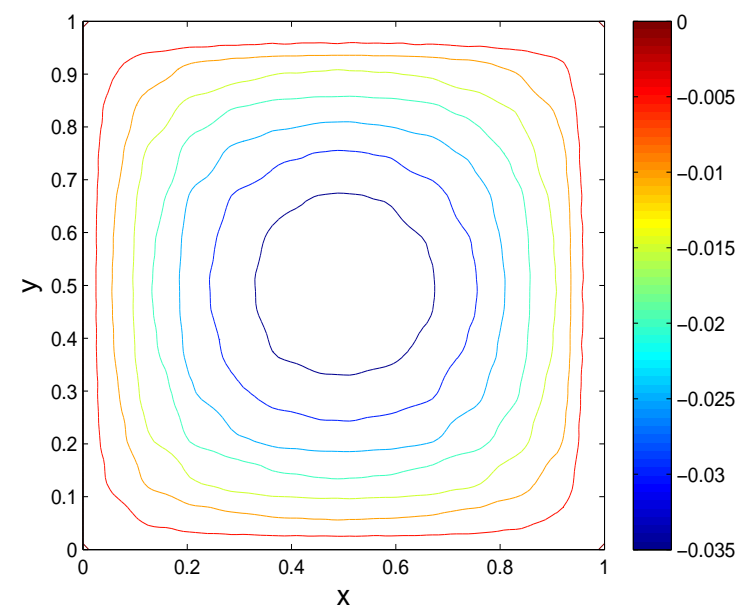

FIGURE 4.6.3. The numerical solution of Example 4.6 (contour plot)

The numerical results of Example 4.5 are shown in Figures 4.5.2-4.5.6 and Table 4.5-4.6. Figure 4.5.2 shows the surface plot of $P_{1}^{\epsilon}(\mathbf{x})$, a typical local basis function on the local cell (with $\mathrm{N}=10$ and $\mathrm{M}=80$ ), which is obtained by the Fourier approximation on the boundary. We point out that in (3.9) the basis function $P_{1}^{\epsilon}(\mathbf{x})$ is defined on a circular domain. However, considering the overlap of the adjacent circular domains, we only need to save the basis function in the square domain surrounded by the sides $\mathbf{x}_{1} \mathbf{x}_{2}, \mathbf{x}_{2} \mathbf{x}_{3}, \mathbf{x}_{3} \mathbf{x}_{4}$, and $\mathbf{x}_{4} \mathbf{x}_{1}$. From Figure 4.5.2 one can find that the local basis function contains the fine scale structure of the multiscale elliptic problem.

In Figure 4.5.3, we show the numerical solution obtained by the Fourier approx- 

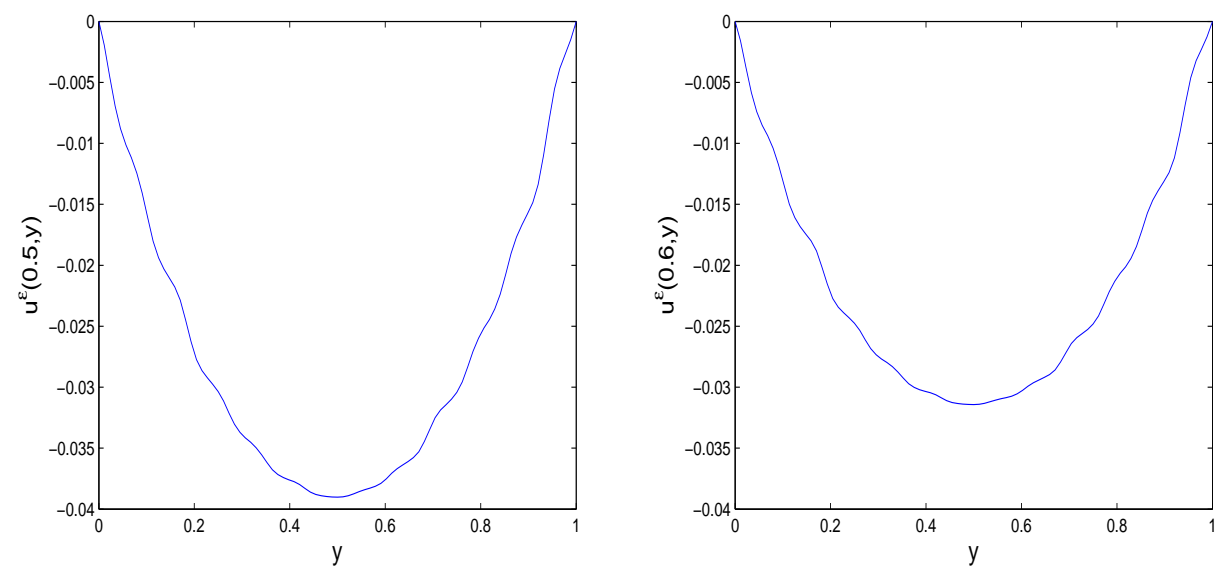

FiguRE 4.6.4. The numerical solutions for Example 4.6 (slice plot)

imation on the boundary (with $N=80$ and $M=40$ ). Figure 4.5.4 gives the corresponding contour plot. Figure 4.5.5 plots the slice solution of $u^{\epsilon}(x, y)$ at $x=0.5$ and $x=0.7$. From these results, we can see that our method can capture the detailed information of the multiscale solution on a coarse mesh grid. From Table 4.5 and 4.6, we find the multiscale tailored finite point methods (MsTFPM) based on Fourier approximation and Lagrange interpolation approximation on the boundary have the same convergence performance in both the $L^{\infty}$ and $L^{2}$ norms. Here the reference solution is obtained on a fine mesh with $\mathrm{N}=80$ and $\mathrm{M}=40$.

EXAmple 4.6. Consider the two dimensional multiscale elliptic problem (4.2) with

$$
\begin{aligned}
& a^{\epsilon}(x, y)=\frac{1}{\left(2+P \sin \left(\frac{2 \pi x}{\epsilon}\right)\right)\left(2+P \sin \left(\frac{2 \pi y}{\epsilon}\right)\right)}, \\
& b^{\epsilon}(x, y)=0, \quad f^{\epsilon}(x, y)=-1,
\end{aligned}
$$

where $\epsilon=0.01$ and $P=1.99$. In this example, the coefficient $a^{\epsilon}(x, y)$ is periodic in $\epsilon$ in each direction with high contrast (defined by $\frac{\max a^{\epsilon}(x, y)}{\min a^{\epsilon}(x, y)}$ ). In the Figure 4.6.1, we only give the surface plot and contour plot of the coefficient $a^{\epsilon}(x, y)$ on a small domain $[0,0.1] \times[0,0.1]$ for demonstration.

The numerical results of Example 4.6 are shown in Figure 4.6.2-4.6.4 and Table 4.7-4.8. In Figure 4.6.2, we show the numerical solutions obtained by the Fourier approximation on the boundary (with $N=80$ and $M=40$ ). Figure 4.6.3 gives the corresponding contour plot. Figure 4.6.4 plots the slice solution of $u^{\epsilon}(x, y)$ at $x=0.5$ and $x=0.6$. From these results, we see that our method can capture the detailed information of the multiscale solution on a coarse mesh grid. In Tables 4.7 and 4.8, we find the multiscale tailored finite point methods (MsTFPM) based on the Fourier approximation and Lagrange interpolation approximation on the boundary have the same convergence performance in both the $L^{\infty}$ and $L^{2}$ norms. Here the reference solution is obtained on fine mesh with $\mathrm{N}=80$ and $\mathrm{M}=40$. 


\section{Conclusion}

We have successfully developed a multiscale tailored finite point method (MsTFPM) for solving the multiscale elliptic problems with rough or highly oscillatory coefficients both in one- and two-dimensions. Our method has been tailored to some particular properties of the multiscale problem, so that it can capture the information of the multiscale solutions on coarse meshes without resolving the fine scale structure. For the one-dimensional problems, we proposed two different types of numerical schemes and proved the stability of the schemes. For the two-dimensional problems, we introduced a boundary approximation technique to impose boundary conditions for the local cell problems. We give two types of numerical schemes based on the Fourier approximation and Lagrange interpolation approximation on the boundaries. Our numerical experiments give convincing evidence that the multiscale tailored finite point method is capable of capturing the large scale solution without resolving the small scale details. An extension of the convergence analysis of the multiscale tailored finite point method for the two-dimensional problems will be our further consideration.

Acknowledgement. This work is supported by the National Natural Science Foundation of China (Grant No. 10971116).

\section{REFERENCES}

[1] R. Ababou, D. McLaughlin, and L.W. Gelhar, Numerical simulation of three-dimensional saturated flow in randomly heterogeneous porous media, Transport in Porous Media 4, 549-565, 1989.

[2] A.F.B. Tompson, Numerical-simulation of chemical migration in physically and chemically heterogeneous porous-media, Water Resour. Res., 29, 3709-3726, 1993.

[3] Y.R. Efendiev and T.Y. Hou, Multiscale finite element methods for porous media flows and their applications, Appl. Numer. Math., 57, 577-596, 2007.

[4] I. Babuska and J. Osborn, Generalized finite element methods: Their performance and their relation to mixed methods, SIAM J. Numer. Anal., 20, 510-536, 1983.

[5] I. Babuska, G. Caloz, and J. Osborn, Special finite element methods for a class of second order elliptic problems with rough coefficients, SIAM J. Numer. Anal., 31, 945-981, 1994.

[6] B. Engquist and E. Luo, New coarse grid operators for highly oscillatory coefficient elliptic problem, J. Comput. Phys., 129, 296-306, 1996.

[7] B. Engquist and E. Luo, Convergence of a multigrid method for elliptic equations with highly oscillatory coefficients, SIAM J. Numer. Anal., 34, 2254-2273, 1997.

[8] T.Y. Hou and X.H. Wu, A multiscale finite element method for elliptic problems in composite materials and porous media, J. Comput. Phys., 134, 169-189, 1997.

[9] T.Y. Hou, X.H. Wu, and Z. Cai, Convergence of a multiscale finite element method for elliptic problems with rapidly oscillating coefficients, Math. Comput., 68, 913-943, 1999.

[10] Z. Chen and T.Y. Hou, A mixed multiscale finite element method for elliptic problems with oscillating coefficients, Math. Comput., 72, 541-576, 2002.

[11] Y.R. Efendiev, T.Y. Hou, and X.H. Wu, The convergence of nonconforming multiscale finite element methods, SIAM J. Numer. Anal., 37, 888-910, 2000.

[12] W. E and B. Engquist, The heterogeneous multiscale methods, Commun. Math. Sci., 1, 87-132, 2003.

[13] W. E, B. Engquist, X.T. Li, W. Ren, and E. Vanden-Eijnden, Heterogeneous multiscale methods: A review, Commun. Comput. Phys., 2, 367-450, 2007.

[14] Y.R. Efendiev and T.Y. Hou, Multiscale Finite Element Methods: Theory and Applications, Springer, 2009.

[15] W. Wang, J. Guzman, and C.-W. Shu, The multiscale discontinuous Galerkin method for solving a class of second order elliptic problems with rough coefficients, J. Numer. Anal. Model., 8, 28-47, 2011.

[16] H. Han, Z. Huang, and B. Kellogg, A Tailored finite point method for a singular perturbation problem on an unbounded domain, J. Sci. Comput., 36, 243-261, 2008. 
[17] H. Han and Z. Huang, Tailored finite point method for a singular perturbation problem with variable coefficients in two dimensions, J. Sci. Comput., 41(2), 200-220, 2009.

[18] Z. Huang, Tailored finite point method for the interface problem, Netw. Heter. Med., 4, 91-106, 2009.

[19] H. Han and Z. Huang, A tailored finite point method for the Helmholtz equation with high wave numbers in heterogeneous medium, J. Comput. Math., 26, 728-739, 2008.

[20] H. Han and Z. Huang, Tailored finite point method for steady-state reaction-diffusion equations diffusion equations, Commun. Math. Sci., 8(4), 887-899, 2010.

[21] M. Protter and H. Weinberger, Maximum Principles in Differential Equations, Prentice-Hall, Englewood Cliffs, New Jersey, 1967.

[22] K. Morton and D. Mayers, Numerical Solution of Partical Differential Equations, Cambridge University Press, 2005.

[23] S. Larsson and V. Thomee, Partical Differential Equations with Numerical Methods, SpringerVerlag, Berlin, Heidelberg, 2003. 